\title{
MATHEMATIKA
}

A JOURNAL OF PURE AND APPLIED MATHEMATICS

$\begin{array}{lll}\text { Vol. 41, Part } 1 & \text { June, } 1994 & \text { No. } 81\end{array}$

\section{ON THE NONLINEAR GROWTH OF SINGLE THREE-DIMENSIONAL DISTURBANCES IN BOUNDARY LAYERS}

\author{
F. T. SMITH, P. A. STEWART AND R. G. A. BOWLES
}

Summary. Experiments indicate the importance of three-dimensional action during transition, while high-Reynolds-number-flow theory indicates a multi-structured type of analysis. In line with this, the three-dimensional nonlinear unsteady triple-deck problem is addressed here, for slower transition. High-amplitude/high-frequency properties show enhanced disturbance growth occurring downstream for single nonlinear oblique waves inclined at angles greater than $\tan ^{-1} \sqrt{2}\left(\approx 54.7^{\circ}\right)$ to the free stream, in certain interesting special cases. The three-dimensional response there is very 'spiky' and possibly random, with sideband instabilities present. A second nonlinear stage, and then an Euler stage, are entered further downstream, although faster transition can go straight into these more nonlinear stages. More general cases are also considered. Sideband effects, sublayer bursting and secondary instabilities are discussed, along with the relation to experimental observations.

\$1. Introduction. There is strong experimental evidence that in general three-dimensional effects ultimately play a large part in transition to turbulence in boundary layers. This is so whether the transition is of the slower type following a linear instability or the faster by-pass type, see [1-5]. Experiments on slower transition, for example, show the original laminar boundary layer [Blasius flow, say] first being altered in a predominantly two-dimensional way, just beyond the neutral position corresponding to the lower branch, and just as linear Tollmien-Schlichting theory predicts, but then three-dimensionality tends to come into play downstream apparently as the pre-cursor of transition even further beyond. (An exception is in the experiments in [37].) This is if 
the imposed level of disturbance amplitudes upstream is not sufficiently small. Otherwise, for sufficiently small disturbances, the flow remains essentially planar and stabilizes further downstream on crossing the upper-branch neutral position.

A number of suggestions have been put forward for theoretically explaining the three-dimensional slower-transition phenomenon. These include most notably resonant triads $[7,23]$, secondary instabilities, associated with Squire modes [6], and wave-mean flow interactions [34], among others; and some experimental evidence exists supporting these interesting suggestions of nonlinear three-dimensional instability, depending on the input. Our aim in this work is to keep the required three-dimensional nonlinear interaction theory on a more rational footing, partly in order to shed more light if possible on the theoretical suggestions above which have often been counched in rather ad hoc terms so far, and partly to discover whether alternative forms of significant and perhaps more powerful three-dimensional behaviour can occur. Threedimensional work already in this area includes that of $[23,34,36]$. In addition it is believed that rational theory can lead to much fuller understanding of the important more nonlinear stages arising subsequently in the transition process, both for slower and faster transitions. See for example the strongly nonlinear theory and comparisons in $[35,36]$. The planar-flow theory investigated earlier $[8,9]$ certainly supports this belief: see also the qualitative and quantitative comparisons with experimental findings and modelling referred to in the next section and recent comparisons between experiments and three-dimensional theory in $[34,36,37]$.

The nonlinear three-dimensional flow theory here assumes throughout that the characteristic Reynolds number $\operatorname{Re} \equiv u_{D} l_{D} / v_{D}$ is large, with $u_{D}, l_{D}, v_{D}$ respectively being the local free-stream speed, the development length of the original boundary layer, and the kinematic viscosity of the incompressible fluid. The assumption that $\mathrm{Re} \gg 1$ throughout provides the only route to a rational theory. Our concern below is then with the behaviour of three-dimensional nonlinear narrow-band wave packets produced downstream, in an originally planar Blasius boundary layer on a flat plate, by means of a nearly-fixedfrequency forcing present upstream, modelling the effects of a vibrating ribbon or free-stream turbulence for example. Further studies have been conducted for the resonant-triad [23] and other types of three-dimensional interaction possible, and for three-dimensional and other basic flows. In all these cases the beginnings of slower transition, and certain faster by-pass transitions discussed later, may be described by the three-dimensional nonlinear unsteady triple-deck problem, illustrated in Fig. 1. There the three-dimensional boundary-layer equations

$$
\begin{gathered}
\partial U / \partial X+\partial V / \partial Y+\partial W / \partial Z=0 \\
\partial U / \partial T+U \partial U / \partial X+V \partial U / \partial Y+W \partial U / \partial Z=-\partial P / \partial X+\partial^{2} U / \partial Y^{2} \\
\partial W / \partial T+U \partial W / \partial X+V \partial W / \partial Y+W \partial W / \partial Z=-\partial P / \partial Z+\partial^{2} W / \partial Y^{2},
\end{gathered}
$$




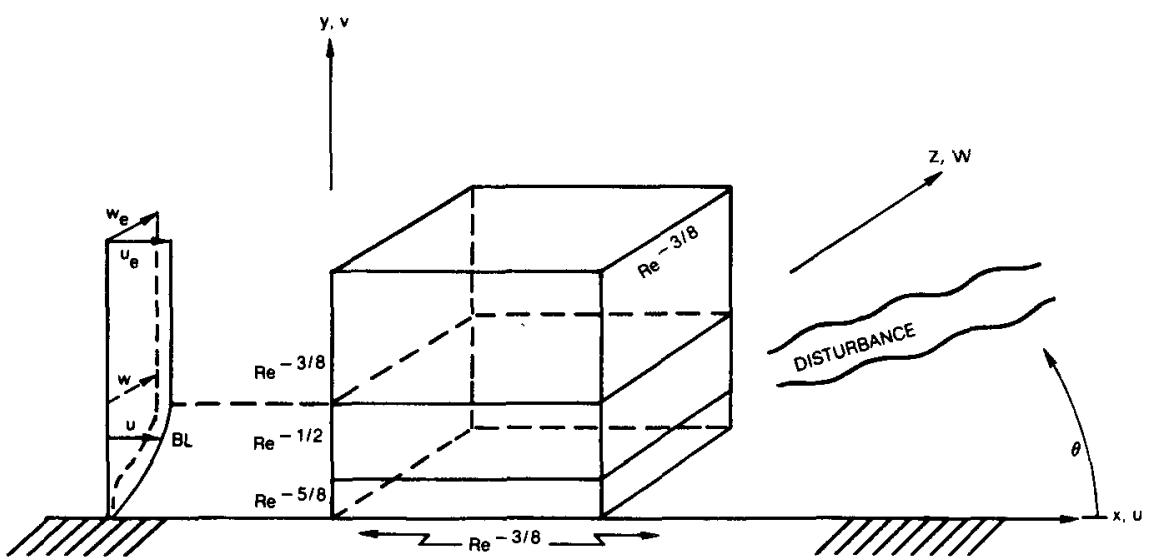

Figure 1. The triple-deck structure, for an incoming two- or three-dimensional boundary layer subjected to a three-dimensional disturbance at a typical oblique angle $\theta$ to the $x$-direction.

apply locally within the boundary layer (in the lower deck, [13]), subject to the main boundary conditions of no slip at the plate surface $(Y=0)$ and of interaction between the unknown pressure $P(X, Z, T)$ and the unknown displacement decrement $A(X, Z, T)$ :

$$
\begin{array}{r}
U=V=W=0 \quad \text { at } \quad Y=0, \\
U \sim Y+A(X, Z, T), \quad W \rightarrow 0, \quad \text { as } \quad Y \rightarrow \infty,
\end{array}
$$

and

$$
P(X, Z, T)=\frac{-1}{2 \pi} \int_{-\infty}^{\infty} \int_{-\infty}^{\infty} \frac{\left(u_{e} \partial / \partial \xi+w_{e} \partial / \partial \eta\right)^{2} A(\xi, \eta, T) d \xi d \eta}{\left[(X-\xi)^{2}+(Z-\eta)^{2}\right]^{1 / 2}}
$$

Here the interaction law (1.1f), holding for local subsonic/incompressible-fluid flow, stems from the potential-flow properties in the upper deck outside the original boundary layer, that is, from the solution of

$$
\left(\frac{\partial^{2}}{\partial X^{2}}+\frac{\partial^{2}}{\partial \bar{y}^{2}}+\frac{\partial^{2}}{\partial Z^{2}}\right) \bar{p}=0
$$

with

$$
\left(\bar{p}, \frac{\partial \bar{p}}{\partial \bar{y}}\right)=\left[P,\left(u_{e} \frac{\partial}{\partial X}+w_{e} \frac{\partial}{\partial Z}\right)^{2} A\right] \text { at } \bar{y}=0 .
$$

Boundedness conditions in $X$ and $\bar{y}$ are also implicit throughout (1.1). In (1.1f, g), for future reference, the constants $u_{e}, w_{e}$ denote the non-dimensional edge velocities of the original $O\left(\mathrm{Re}^{-1 / 2}\right)$ boundary layer (in a suitably aligned cartesian frame) if the latter is three-dimensional, although virtually all the analysis below addresses the case of the planar original boundary layer for which $u_{e}=1, w_{e}=0$. In addition (see also Fig. 1) the Cartesian coordinates 
$(x, y, z)$, the corresponding velocity components $(u, v, w)$, the pressure $p$ and the time $t$ used are nondimensionalized with respect to $l_{D}, u_{D}, \rho_{D} u_{D}^{2}, l_{D} u_{D}^{-1}$ in turn, with $\rho_{D}$ standing for the fluid density. The scalings behind the local nonlinear governing equations and constraints $(1.1 \mathrm{a}-\mathrm{f})$ then have the form

$$
\begin{aligned}
& {[u, v, w, p]=\left[\lambda^{1 / 4} \operatorname{Re}^{-1 / 8} U, \lambda^{3 / 4} \operatorname{Re}^{-3 / 8} V, \lambda^{1 / 4} \operatorname{Re}^{-1 / 8} W, \lambda^{1 / 2} \operatorname{Re}^{-1 / 4} P\right],} \\
& {\left[x-x_{0}, y, z, t\right]} \\
& \quad=\left[\lambda^{-5 / 4} \operatorname{Re}^{-3 / 8} X, \lambda^{-3 / 4} \operatorname{Re}^{-5 / 8} Y, \lambda^{-5 / 4} \operatorname{Re}^{-3 / 8} Z, \lambda^{-3 / 2} \operatorname{Re}^{-1 / 4} T\right],
\end{aligned}
$$

while in (1.1g), which is an alternative to (1.1f), $p \propto \operatorname{Re}^{-1 / 4} \bar{p}$ and $y \propto \operatorname{Re}^{-3 / 8} \bar{y}$. The streamwise and spanwise length scales in the triple-deck box around the station $x=x_{0}$ are therefore large compared with the original $O\left(\mathrm{Re}^{-1 / 2}\right)$ boundary-layer thickness but small compared with the development $l_{D}$. Moreover, the streamwise and spanwise velocities $u, w$ in the thin viscous lower deck are comparable in magnitude, although the $W$-profile has a jet-like form whereas $U$ is affected by the basic-flow shear, in (1.1e). The origin shift $x_{0}$ in (1.2b) allows for movement of the local flow structure upstream or downstream and it affects matters only through its control of the original Blasius skin friction factor $\lambda\left(x_{0}\right) \equiv \hat{\lambda} x_{0}^{-1 / 2}(\hat{\lambda}=0 \cdot 332)$, appearing in (1.2). This control is important nonetheless.

The governing equations (1.1), which are independent of the Reynolds number $\operatorname{Re}$ and the position $x_{0}$ (or the factor $\lambda\left(x_{0}\right)$ ), are derived rationally from the Navier-Stokes equations under the scalings (1.2). They are also related closely, in their linearized version, with classical work on the OrrSommerfeld equation for infinitesimal disturbances at increasing $\mathrm{Re}$, near the lower branch of the neutral curve, as $[11,13]$ mention. In the linear version, where $(U, V, W, P, A)$ are considered as small perturbations of the original boundary-layer-flow solutions $(Y, 0,0,0,0),(1.1)$ yields the dispersion relation

$$
\begin{gathered}
\hat{\alpha}^{5 / 3} \operatorname{Ai}^{\prime}\left(\xi_{0}\right)=e^{i \pi / 6}\left(\hat{\alpha}^{2}+\hat{\beta}^{2}\right)^{1 / 2}\left(u_{e} \hat{\alpha}+w_{e} \hat{\beta}\right)^{2} \int_{\xi_{0}}^{\infty} \operatorname{Ai}(q) d q, \\
\text { where } \xi_{0} \equiv \Omega e^{-5 i \pi / 6} \hat{\alpha}^{-2 / 3},
\end{gathered}
$$

with Ai denoting the Airy function. This is for small waves proportional to $\exp (i \hat{\alpha} X+i \hat{\beta} Z-i \Omega T)$ having $O(1)$ streamwise and spanwise scaled wavenumbers $\hat{\alpha}, \hat{\beta}$ and effective frequency $\Omega$. The role of $u_{e}, w_{e}$ in $(1.3)$ is of interest $[15,16,38]$, although here we consider $u_{e}=1, w_{e}=0$. The neutral case $(\hat{\alpha}, \hat{\beta}$, $\Omega$ all real) then occurs for a finite value of the effective frequency $\Omega$ which in the planar case is $\Omega=\Omega_{N}=2 \cdot 30$ (approx., [11]), consistent with Orr-Sommerfeld asymptotes. So, in real terms, for an upstream imposed disturbance of prescribed dimensional frequency $\Omega_{D} \approx u_{D} \partial / \partial\left(l_{D} t\right)$ say, the linear version predicts the planar-flow neutral position $x_{0}=x_{0 N}$ to be $x_{0}=x_{0 N}=$ $\left(\Omega_{N} u_{D} / \Omega_{D} l_{D}\right)^{4 / 3} \hat{\lambda}^{2} \mathrm{Re}^{1 / 3}$ to leading order, because of the underlying factor $\lambda\left(x_{0}\right)$ in (1.2). See experimental comparisons in Section 2. Downstream of this lowerbranch position, for stations $x_{0}>x_{0 N}$, the skin friction factor $\lambda\left(x_{0}\right)$ then decreases and in consequence the effective $\Omega$ acting in (1.3) increases, forcing linearized disturbance growth to take place, that is, $\operatorname{Im}(\Omega)$ positive with 
$\hat{\alpha}, \hat{\beta}$ real for temporal growth or $\operatorname{Im}(\hat{\alpha})$ negative with $\Omega, \hat{\beta}$ real for streamwise growth, again in line with experiments.

Our concern is with what happens nonlinearly, once Tollmien-Schlichting disturbance growth starts in the above fashion. The full nonlinear threedimensional system (1.1) then comes into operation, and in our view (1.1) undoubtedly represents a significant nonlinear step in the ensuing transition process, for sizeable disturbances. It is a step requiring fairly large-scale computation in general, however. (See also [35, 39-41]). The present work pursues the alternative of a large-amplitude analysis of (1.1). This is associated with the regime of high effective frequencies $\Omega(\approx|\partial / \partial T|$ in $(1.1)$ ), thus representing increased distances $x_{0}$ downstream, in view of the $\lambda$ factors in (1.2), as is desirable on practical grounds. The high-frequency regime also represents the effects of abruptly increased disturbance amplitudes and frequencies, by-passing the earlier finite- $\Omega$ regime due to relatively sudden sizeable free-stream unsteadiness becoming present for instance; that is, it also models the type of faster transition process referred to earlier on. Again, as noted in $[8,9]$ (see also $[12,42]$ ), the high-frequency regime is a broad theoretical one, holding in fact for the range $1 \ll \Omega \leqslant O\left(\mathrm{Re}^{1 / 4}\right)$ (or dimensional frequencies $\Omega_{D}$ satisfying $u_{D} l_{D}^{-1} \mathrm{Re}^{1 / 4} \ll \Omega_{D} \leqslant u_{D} l_{D}^{-1} \mathrm{Re}^{1 / 2}$ approximately) and enabling a more analytical study of initial-value problems for nonlinear two- or three-dimensional wave packets.

In Section 2 below, the two-dimensional theory and its relevance to experimental observations are retraced, to set the scene. In particular, the highfrequency theory outlined there (from $[8,9]$ ) points to the existence of at least three stages in the downstream development. Section 3 then addresses the first such stage encountered, stage 1 , but for a single three-dimensional nonlinear disturbance, evolving from a three-dimensional wave with, to leading order, prescribed spanwise wavenumber and frequency at the input station upstream. This is in contrast with the two or more waves active in other interactions, e.g., $[23,43]$, where a relatively fine tuning of the input is sometimes necessary. The controlling equation arrived at in Section 3 is studied numerically and analytically in Sections 4 and 5 for an intriguing and potentially important special case of oblique waves, for which a Ginzburg-Landau-like form holds. A type of sideband instability is found to occur for oblique waves inclined at angles $\theta>\theta_{\text {crit }}$ from the free-stream direction,

$$
\theta_{\text {crit }}=\tan ^{-1}(\sqrt{ } 2) \approx 54 \cdot 7^{\circ} \text {. }
$$

The instability is fast-growing, of the kind $\exp (\exp ($ time $))$. At large times or distances downstream it leads to a strong nonlinear growth, much faster than in linear theory, and with a degree of apparent randomness present, as opposed to the planar or $\theta<\theta_{\text {crit }}$ disturbances which are relatively benign in this first stage and grow more slowly than in linear theory. The ensuing breakdown to stage 2, followed by the Euler stage, both of which are more nonlinear, is reached sooner if $\theta>\theta_{\text {crit }}$, with the fastest breakdown taking place exactly for $\theta=\theta_{\text {crit }}$ in (1.4). This is discussed in Section 7, after a discussion of more general cases in Section 6. Further comments are presented in Section 8, including sublayer bursting, secondary instabilities and qualitative comparisons with recent experiments, which also suggest the appearance of sideband instability 
as well as indicating significant three-dimensional action at oblique angles reasonably near to the value in (1.4).

\$2. Briefly reviewing the two-dimensional theory. Since the underlying flow of concern is a boundary layer, which is itself necessarily a high-Reynoldsnumber limit, the only way [11] strictly to develop a rational self-consistent theory is to assume throughout that $\mathrm{Re} \gg 1$, for both the original motion and the nonlinearly disturbed motion. That being so, we are led immediately to unsteady triple-deck theory, for which the three-dimensional governing equations are given in (1.1), as far as nonlinear lower-branch properties are concerned. The three-dimensional flow properties are studied further in Sections 3-7 below. In the two-dimensional case, reviewed in this section, the tripledeck interaction is controlled by the planar boundary-layer equations and constraints obtained from setting $W, w_{e}, \partial / \partial Z$ to zero in (1.1). This applies to the nonlinear development of free disturbances in an otherwise attached boundary layer, or to forced disturbances, with suitable alterations in the boundary conditions to accommodate wall effects or free-stream turbulence for instance. In addition, the influence of an applied pressure gradient is felt through its alteration of the skin friction factor $\lambda\left(x_{0}\right)$ in (1.2), with an adverse or favourable pressure gradient thereby producing an advance or delay, respectively, in the Tollmien-Schlichting instabilities.

As a check on the physical relevance of the rational two-dimensional theory, and as a reminder, it is noted first that the linearized version agrees well with the experimental measurements for small disturbances in a Blasius boundary layer, particularly concerning nonparallel flow effects: see [11], also [24]. A further boost here is given by [12]'s interesting comparison between the linearized theory and experiments on non-flat surfaces. Second, weakly nonlinear properties from the two-dimensional theory, near the critical frequency, are in close agreement with the corresponding Navier-Stokes-based computations and experiments: see $[11,18]$. Next, a composite computational approach based on the theory and applied for finite Reynolds numbers is found to compare very favourably with Navier-Stokes computations even at low subcritical Reynolds numbers: see [17]. Finally, a large-amplitude analysis $[8,9]$ corresponding to high typical frequencies $\Omega=O[|\partial / \partial T|]$ and shortened streamwise length scales, in the planar-flow version of (1.1), shows the nonlinearly disturbed unsteady motion passing through two stages in its progress downstream. In the first stage, 1, nonlinearity has a first chance to affect the lineardisturbance growth but is found to fail to do so substantially, although the spatial spreading is greatly enhanced. In the subsequent stage 2 inviscid nonlinear dynamics tend to dominate most of the lower-deck flow, leading to the Benjamin-Ono equation for the displacement function $-A(X, T)$,

$$
\frac{\partial A}{\partial T}+A \frac{\partial A}{\partial X}=-\frac{1}{\pi} \int_{-\infty}^{\infty} \frac{\partial^{2} A}{\partial \xi^{2}} \frac{d \xi}{(X-\xi)}
$$

from (1.1), but this is subject to the response of a viscous nonlinear sublayer closer to the surface. See also [44] and the encouraging theoreticalexperimental comparisons in [37]. The viscous sublayer can readily force spikelike eruptions of vorticity into the inviscid region [8], eventually, one could 
expect, in a fairly random fashion. This, coupled with the secondary instabilities of the Rayleigh kind which are possible [27], and with recent computations of the full system $(1.1)$ in the two-dimensional version $[9,10]$, forms an encouraging connection both with planar-flow calculations of the Navier-Stokes equations [26] and with certain experimental observations on transitional or turbulent boundary layers: see $[8,25]$. The connection with experiment seems to be enhanced, moreover, when a further theoretical stage bringing in the Euler equations is studied, this being associated either with the natural development of a free nonlinear disturbance downstream of stages 1,2 or with the abrupt forcing from higher-frequency large disturbances which can by-pass the previous stages 1,2 . There the Euler equations hold across the entire boundary layer apart from a classical viscous sublayer which, as above, sends vorticity bursts into the outer unsteady flow [45]. Sublayer bursting is a well-known major part of the make-up of a turbulent boundary layer, as recent work in $[40,41,45,46]$ emphasizes.

The two-dimensional theory so far, then, produces an encouraging resemblance to many practical aspects of boundary-layer transition as well as to wholly numerical planar-flow results. It acts overall as a very useful starting point and a test-bed for ideas and for the development of computational schemes, and it also provides suggestions for numerical methods at finite Reynolds numbers. Given that start, we turn now to the three-dimensional nonlinear theory since experiments and computational work indicate that threedimensionality usually becomes very important as nonlinear disturbances amplify. Certain of the above features carry through to three dimensions but there are some very notable exceptions which are potentially of practical significance.

§3. Three-dimensional nonlinear properties. The nonlinear threedimensional unsteady triple-deck problem to be addressed is posed in (1.1), with the Reynolds number and the local skin friction factor scaled out explicitly in (1.2). Linear and weakly nonlinear properties are described in [13] for the case of a planar basic boundary layer flow, $w_{e}=0$, and we concentrate primarily on that case here, leaving discussion of three-dimensional starting flows with $w_{e} \neq 0$ to further study.

Computations are required for the all-important nonlinear threedimensional regime of (1.1) in general and we believe these should prove of much interest. For example, according to [13], subcritical as well as supercritical weakly nonlinear states are possible when several three-dimensional modes interact, at a finite frequency $\Omega \equiv O[|\partial / \partial T|]$, while [27] suggests short-scale instabilities may occur in the nonlinear regime. Further, the present work and its companion, [23], indicate a number of distinctly three-dimensional features arising at high frequencies, which appear to have rather close connection with experimental findings and add weight to the physical and theoretical interest in the nonlinear system (1.1).

Our concern, then, is in the high-frequency range where $\Omega$ is large, and this is for three main reasons. First, even in the two-dimensional case the highfrequency range yields properties which, by means of the successive stages $(1,2$, Euler) referred to in Section 2, resemble many of those occurring experimentally in boundary-layer transition. See also the recent comparisons in [37]. 
Second, the high-frequency range shows quite clearly how transition, via enhanced nonlinearity, can take place either through an increase in the downstream distance $x_{0}$, by affecting the skin friction factor $\lambda$ in (1.2), or through an abrupt raising of the dominant frequencies present in the disturbance. The third reason is the analytical one that, apart from this high-frequency range, any nonlinear initial-value problem for (1.1) sets a completely numerical task. In what follows we address stage 1 , as a start, to examine whether for a single wave packet three-dimensionality makes much difference, or not, from the planar case studied before.

Considering large $\Omega$, therefore, we have the multiple scalings

$$
\begin{gathered}
\partial / \partial T \rightarrow \Omega \partial / \partial T_{0}+\Omega^{1 / 2} \partial / \partial T_{1}+\partial / \partial T_{2}+\ldots \\
\partial / \partial X \rightarrow \Omega^{1 / 2} \partial / \partial X_{0}+\partial / \partial X_{1}+\Omega^{-1 / 2} \partial / \partial X_{2}+\ldots \\
\partial / \partial Z \rightarrow \Omega^{1 / 2} \partial / \partial Z_{0}+\partial / \partial Z_{1}+\Omega^{-1 / 2} \partial / \partial Z_{2}+\ldots
\end{gathered}
$$

Most of the scalings here and below are implied by extension of the earlier two-dimensional theory and by appeal to the large- $\Omega$ version of the linearizedstability eigenrelation (1.3). Unlike the planar case, however, it is helpful here to consider two or three scales in the normal direction $Y$ also (Fig. 2), one being for the viscous Stokes layer I where $Y$ is small and $O\left(\Omega^{-1 / 2}\right)$, studied in Sub-Section 3.1 below, and another being for the large, $O(\Omega)^{1 / 2}$, outer inviscid region II which houses the critical layer and is discussed in Sub-Section 3.2. A third region is a buffer layer III for the mean-flow correction found below. Both regions I, II play a strong part (Sub-Section 3.3) in the development of the nonlinear disturbance. The unknown pressure and displacement distributions have the forms

$$
\begin{aligned}
& P=\Omega^{1 / 2} P_{0}+P_{1}+\Omega^{-1 / 2} P_{2}+\ldots, \\
& A=A_{0}+\Omega^{-1 / 2} A_{1}+\Omega^{-1} A_{2}+\ldots,
\end{aligned}
$$

throughout the current stage 1 .

Sub-Section 3.1. The Stokes Layer. In the viscous Stokes layer I, the flow solution expands according to

$$
\begin{gathered}
U=U_{0}+\Omega^{-1 / 2} U_{1}+\Omega^{-1} U_{2}+\ldots \\
V=V_{0}+\Omega^{-1 / 2} V_{1}+\Omega^{-1} V_{2}+\ldots \\
W=W_{0}+\Omega^{-1 / 2} W_{1}+\Omega^{-1} W_{2}+\ldots
\end{gathered}
$$

with $Y=\Omega^{-1 / 2} \tilde{y}$ and $\tilde{y}$ is $O(1)$. It is observed that all three components $U, V$, $W$ are comparable in magnitude here. The governing equations resulting from substitution of (3.1)-(3.3) into (1.1) are the three-dimensional counterparts of those in [9] and so here only their successive solutions will be written down. More details are given in Appendix A. 


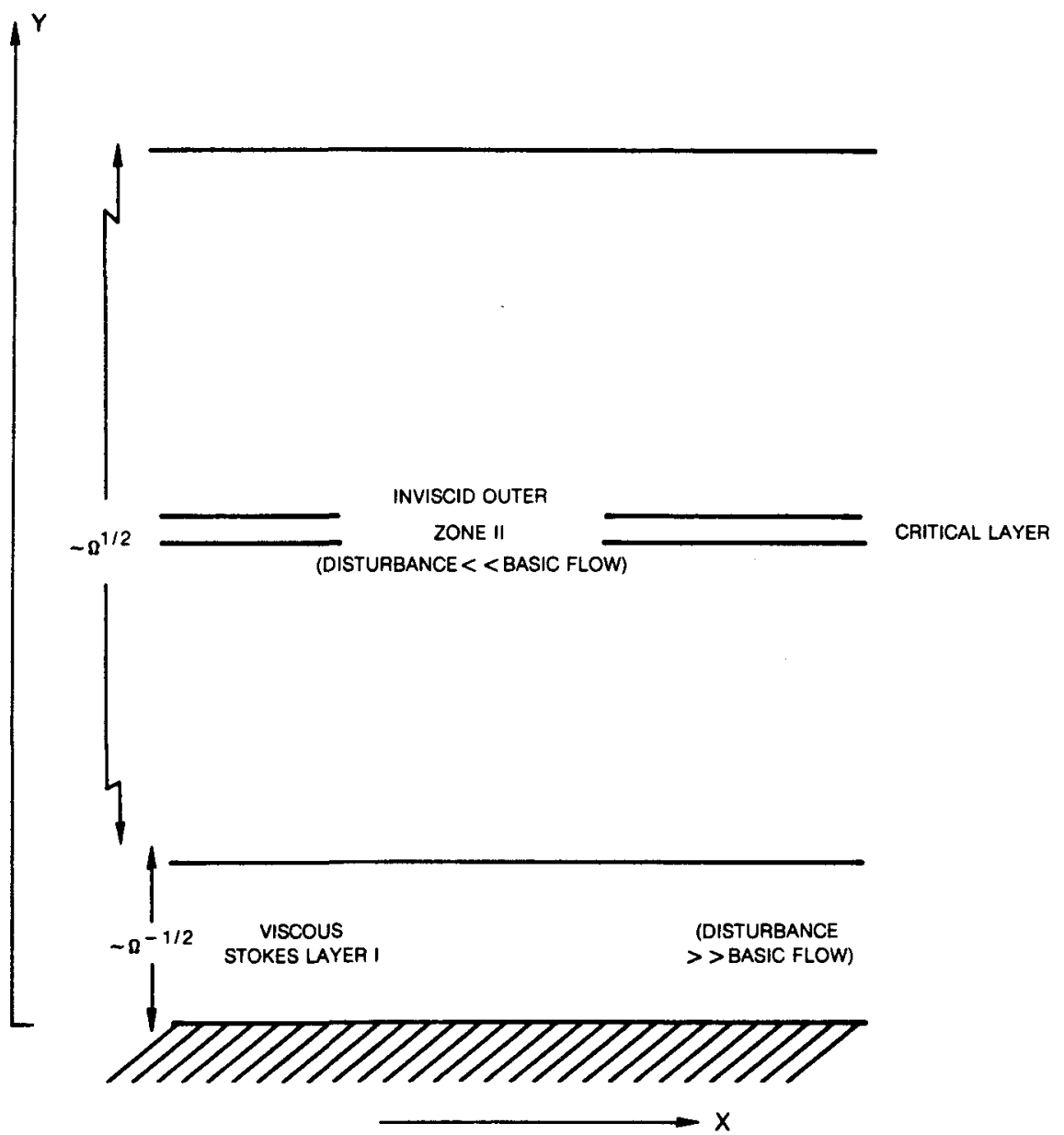

Figure 2. The structure of the lower deck for the high-frequency high-amplitude disturbances considered in Sections 3-6.

The solutions have the form

$$
\begin{gathered}
\left(U_{0}, P_{0}\right)=\left(U_{01}, P_{01}\right) E+\text { c.c. } \\
\left(U_{1}, P_{1}\right)=\left(U_{11}, P_{11}\right) E+\text { c.c. }+\left(U_{12}, P_{12}\right) E^{2}+\text { c.c. }+\left(U_{1 M}, P_{1 M}\right), \\
\left(U_{2}, P_{2}\right)=\left(U_{21}, P_{21}\right) E+\text { c.c. }+ \text { terms in } E^{ \pm 2}, E^{ \pm 3}, E^{0}
\end{gathered}
$$

and similarly for $V_{n}, W_{n}, A_{n}(n=0,1,2)$, where c.c. denotes the complex conjugate of the preceding expression, while the wave-like terms involving

$$
E \equiv \exp \left[i \alpha X_{0}+i \beta Z_{0}-i T_{0}\right]
$$

contain all the dependence on the fastest scales $X_{0}, Z_{0}, T_{0}$. The constants $\alpha(>0)$ and $\beta$ are the streamwise and cross-stream wavenumbers, to be detailed 
later. In the first order terms, the fundamentals in (3.4a),

$$
\begin{aligned}
{\left[U_{01}, W_{01}\right] } & =[\alpha, \beta] P_{01}\left(1-e^{m \tilde{y}}\right), \quad \text { with } \quad m \equiv e^{3 \pi i / 4}, \quad(3.6 \mathrm{a}, \mathrm{b}) \\
V_{01} & =-i\left(\alpha^{2}+\beta^{2}\right) P_{01}\left(\tilde{y}-m^{-1} e^{m \tilde{y}}+m^{-1}\right) .
\end{aligned}
$$

In the second-order terms in $(3.4 \mathrm{~b})$, we find

$$
\begin{gathered}
{\left[U_{12}, W_{12}\right]=\left[D_{12}, C_{12}\right] e^{\sqrt{2} m \tilde{y}}+[\alpha, \beta] P_{12}} \\
+[\alpha, \beta] P_{01}^{2}\left(\alpha^{2}+\beta^{2}\right)\left\{1 / 2+(1+i m \tilde{y}) e^{m \tilde{y}}\right\}, \\
\frac{i}{2} V_{12}=\left(\alpha^{2}+\beta^{2}\right)\left\{\frac{C_{12}}{\sqrt{2} \beta m}\left(e^{\sqrt{2} m \tilde{y}}-1\right)+P_{12} \tilde{y}+\left(\alpha^{2}+\beta^{2}\right) P_{01}^{2} \tilde{y}\left(1 / 2+e^{m \tilde{y}}\right)\right\},
\end{gathered}
$$

for the second harmonic, where

$$
\left[D_{12}, C_{12}\right]=-[\alpha, \beta]\left\{P_{12}+\frac{3}{2} P_{01}^{2}\left(\alpha^{2}+\beta^{2}\right)\right\}
$$

the mean-flow contributions are

$$
\begin{gathered}
U_{1 M}=i\left|P_{01}\right|^{2}\left[\left(\alpha^{2}+\beta^{2}\right) \alpha m^{*}\left\{e^{m^{*} \tilde{y}}\left(\tilde{y} / m^{* 2}-2 / m^{* 3}+1 / m^{*}\right)-e^{-\sqrt{2} \tilde{y}} / 2 m\right\}\right. \\
+\alpha \beta^{2}\left\{e^{m \tilde{y}} / m^{2}+e^{m^{*} \tilde{y}} / m^{* 2}\right\}-\left(\alpha^{2}+\beta^{2}\right) \alpha m^{*}\left\{-2 / m^{* 3}-1 / 2 m+1 / m^{*}\right\} \\
\left.-\alpha \beta^{2}\left\{1 / m^{2}+1 / m^{* 2}\right\}\right]+ \text { c.c. }+\tilde{y}, \\
V_{1 M}=0,
\end{gathered}
$$

with $W_{1 M}$ being as in (3.8a) but subject to the interchange of $\alpha, \beta$ and to the omission of the final contribution $+\tilde{y}$, which comes from the underlying shear in (1.1); and the extra fundamental gives

$$
\left(\alpha U_{11}+\beta W_{11}\right)=\tilde{c}_{11}\left(1-e^{m \tilde{y}}\right)+\tilde{b}_{11} \tilde{y} e^{m \tilde{y}},
$$

where

$$
\begin{aligned}
2 m \tilde{b}_{11} & =-\left(\alpha^{2}+\beta^{2}\right), \\
i \tilde{c}_{11} & =\left(\alpha^{2}+\beta^{2}\right)\left(i P_{11}+\frac{\partial P_{01}}{\partial T_{1}}\right)+\alpha \frac{\partial P_{01}}{\partial X_{1}}+\beta \frac{\partial P_{01}}{\partial Z_{1}} .
\end{aligned}
$$

Here * also denotes the complex conjugate. Finally, the reproduced fundamentals in the third-order terms in (3.4c) are found to yield the properties

$$
U_{21} \sim-\beta^{2} P_{01} \tilde{y}+B_{21}, \quad W_{21} \sim \alpha \beta P_{01} \tilde{y}+C_{21}, \quad \text { as } \quad \tilde{y} \rightarrow \infty,
$$

which are needed for the subsequent matching in Sub-Section 3.3. Here the functions $B_{21}, C_{21}$ satisfy

$$
\begin{gathered}
-i\left(\alpha \beta_{21}+\beta C_{21}\right)+\frac{\partial \tilde{c}_{11}}{\partial T_{1}}+\left(\alpha^{2}+\beta^{2}\right) \frac{\partial P_{01}}{\partial T_{2}}+i\left(\alpha^{2}+\beta^{2}\right) P_{01}\left(\alpha \beta_{1 M}+\beta C_{1 M}\right) \\
-\frac{i \alpha}{m}\left(\alpha^{2}+\beta^{2}\right) P_{01}+\left[P_{12}+\frac{1}{2} P_{01}^{2}\left(\alpha^{2}+\beta^{2}\right)\right] i\left(\alpha^{2}+\beta^{2}\right)^{2} P_{01}^{*} \\
=-i\left(\alpha^{2}+\beta^{2}\right) P_{21}-\left[\alpha \frac{\partial P_{01}}{\partial X_{2}}+\beta \frac{\partial P_{01}}{\partial Z_{2}}\right]-\left[\alpha \frac{\partial P_{11}}{\partial X_{1}}+\beta \frac{\partial P_{11}}{\partial Z_{1}}\right]
\end{gathered}
$$


and $B_{1 M}, C_{1 M}$ are the $O(1)$ functions appearing in the asymptotes of the mean flow,

$$
U_{1 M} \sim \tilde{y}+B_{1 M}, \quad W_{1 M} \rightarrow C_{1 M}, \quad \text { as } \quad \tilde{y} \rightarrow \infty,
$$

so that the induced Stokes-slip velocity combination appearing in $(3.10 \mathrm{~b})$ is given by

$$
\left(\alpha \beta_{1 M}+\beta C_{1 M}\right)=3\left|P_{01}\right|^{2}\left(\alpha^{2}+\beta^{2}\right)^{2},
$$

from $(3.8 \mathrm{a})$.

The above solutions satisfy the no-slip conditions at $\tilde{y}=0$ but do not match directly to the outer boundary conditions in (1.1). For, in particular, $W_{01}$ in (3.6b) tends to $\beta P_{01}$ at large $\tilde{y}$, as opposed to the required outer constraint of zero on the cross-stream velocity $W$ in (1.1). Therefore, unlike in the twodimensional case of $[8,9]$, an outer region II is required here: see next SubSection. Further, many of the three-dimensional features in the above solutions follow from Squires-like transformations, even with nonlinearity present, as in the combinations in (3.9a), (3.10b), (3.11b). Similar combinations apply in the region II studied below. Another point to mention arises in comparing the three-dimensional expansions with those applying in the inviscid water-wave theory of [28]. The latter work raises the possibility of an additional meanpressure contribution, $P_{O M}$ say, in (3.4a). If this is present, however, then $U_{1 M}$ in (3.18a) and $W_{1 M}$ receive extra contributions $\tilde{y}^{2}\left(\partial P_{0 M} / \partial X_{1}, \partial P_{0 M} / \partial Z_{1}\right) / 2$, in turn, which dominate the large- $\tilde{y}$ behaviour. These contributions cannot be matched to the outer constraint in region II below, and so it is necessary that $P_{0 M}=0$, as assumed in (3.4a). Lastly here, it is of interest that, as in the planar theory, the disturbance amplitude in region $I$ is much greater than the total mean flow (which appears first in (3.8a)) ; nevertheless, the nonlinearity is absent from the fundamental and first influences only the second harmonics and the mean flow ( $c f$. the subsequent stage 2); and the induced Stokes slip velocity and displacement arise among the linear and nonlinear effects. The flow solutions above for the Stokes layer I will be matched in Sub-Section 3.3 with those of the outer region II which is discussed next.

Sub-Section 3.2. The Outer Inviscid Region. In the outer inviscid region II, where $Y=\Omega^{1 / 2} \hat{y}$ with $\hat{y}$ of order unity, the flowfield as implied by the behaviour of the Stokes-layer solutions has the expansion

$$
\begin{gathered}
U=\Omega^{1 / 2} \hat{y}+\hat{U}_{0}+\Omega^{-1 / 2} \hat{U}_{1}+\ldots, \\
V=\Omega \hat{V}_{0}+\Omega^{1 / 2} \hat{V}_{1}+\hat{V}_{2}+\ldots, \\
W=\hat{W}_{0}+\Omega^{-1 / 2} \hat{W}_{1}+\ldots
\end{gathered}
$$

So here the basic shear flow provides the dominant velocity. From substitution of $(3.12 \mathrm{a}-\mathrm{c})$, with (3.1), (3.2), into (1.1), we find the successive governing equations given in Appendix A. Their solutions are of the form

$$
\begin{gathered}
\hat{W}_{0}=\hat{W}_{01} E+\text { c.c. } \\
\hat{W}_{1}=\hat{W}_{11} E+\text { c.c. }+\hat{W}_{12} E^{2}+\text { c.c. }+\hat{W}_{1 M}, \\
\hat{W}_{2}=\hat{W}_{21} E+\text { c.c. }+ \text { terms in } E^{ \pm 2}, E^{ \pm 3}, E^{0},
\end{gathered}
$$


and similarly for $\hat{U}_{n}, \hat{V}_{n}(n=0,1,2)$. This basically inviscid region has to produce the change from the inner matching conditions, including

$$
\begin{gathered}
{\left[\hat{U}_{01}, \hat{W}_{01}\right] \rightarrow[\alpha, \beta] P_{01}, \quad \hat{V}_{01} \rightarrow 0,} \\
{\left[\hat{U}_{12}, \hat{W}_{12}\right] \rightarrow[\alpha, \beta]\left\{P_{12}+P_{01}^{2}\left(\alpha^{2}+\beta^{2}\right) / 2\right\}, \quad \hat{V}_{12} \rightarrow 0,} \\
\alpha \hat{U}_{11}+\beta \hat{W}_{11} \rightarrow \tilde{c}_{11}, \quad \hat{V}_{11} \rightarrow 0, \\
{\left[\hat{U}_{21}, \hat{W}_{21}\right] \rightarrow\left[B_{21}, C_{21}\right], \quad \hat{V}_{21} \rightarrow-i\left(\alpha^{2}+\beta^{2}\right) \frac{P_{01}}{m},}
\end{gathered}
$$

for $\hat{y} \rightarrow 0$, to the outer boundary conditions holding for $\hat{y} \rightarrow \infty$ :

$$
\begin{gathered}
{\left[\hat{U}_{01}, \hat{U}_{12}, \hat{U}_{1 M}, \hat{U}_{11}, \hat{U}_{21}\right] \rightarrow\left[A_{01}, A_{12}, A_{1 M}, A_{11}, A_{21}\right],} \\
{\left[\hat{W}_{01}, \hat{W}_{12}, \hat{W}_{1 M}, \hat{W}_{11}, \hat{W}_{21}\right] \rightarrow[0,0,0,0,0] .}
\end{gathered}
$$

Here all the constraints $(3.14 \mathrm{a}-\mathrm{d})$ stem from the Stokes-layer properties, with, most notably, the Stokes displacement influencing the third-order normal velocity $\hat{V}_{21}$ in $(3.14 \mathrm{~d})$. The constraints $(3.14 \mathrm{e}, \mathrm{f})$ are from $(1.1)$. We observe however that the mean-flow terms $\hat{U}_{1 M}, \hat{W}_{1 M}$ do not match directly with $B_{1 M}$, $C_{1 M}$ as $\hat{y}$ tends to zero, because of a buffer layer (III) lying between regions I, II and bringing viscous effects into play for the mean flow. Hence the individual components in (3.13) are found to be as follows. First, to comply with $(3.14 \mathrm{e}, \mathrm{f})$,

$$
\hat{U}_{01}=\frac{\beta^{2} P_{01}}{\alpha(\alpha \hat{y}-1)}+A_{01}, \quad \hat{W}_{01}=\frac{-\beta P_{01}}{(\alpha \hat{y}-1)}, \quad \hat{V}_{01}=-i \alpha A_{01} \hat{y},
$$

with $(3.14 \mathrm{a})$ then giving the pressure-displacement relation

$$
\alpha A_{01}=\left(\alpha^{2}+\beta^{2}\right) P_{01} .
$$

Second, and in similar fashion,

$$
\begin{gathered}
\hat{U}_{12}=\frac{\beta^{2} P_{12}}{\alpha(\alpha \hat{y}-1)}+\frac{P_{01}^{2} \beta^{2}}{2 \alpha}\left(\alpha^{2}+\beta^{2}\right)\left\{\frac{-2}{(\alpha \hat{y}-1)^{2}}-\frac{1}{(\alpha \hat{y}-1)^{3}}\right\}+A_{12}, \\
\alpha \hat{U}_{12}+\beta \hat{W}_{12}=\alpha A_{12}, \\
\hat{V}_{12}=-2 i \alpha A_{12} \hat{y},
\end{gathered}
$$

and $(3.14 b)$ then requires

$$
\alpha A_{12}=\left(\alpha^{2}+\beta^{2}\right)\left\{P_{12}+\frac{1}{2} P_{01}^{2}\left(\alpha^{2}+\beta^{2}\right)\right\} .
$$

Third, $\tilde{V}_{1 M} \equiv 0$ but $\hat{U}_{1 M}, \hat{W}_{1 M}$ (and hence $\hat{Q}_{1 M} \equiv \alpha \hat{U}_{1 M}+\beta \hat{W}_{1 M}$ ) remain unknown at this level: see also Appendix B. Fourth, we have

$$
\alpha \hat{U}_{11}+\beta \hat{W}_{11}\left(\equiv \hat{Q}_{11}\right)=\frac{i \beta}{\alpha} \frac{\left(\beta \partial P_{01} / \partial X_{1}-\alpha \partial P_{01} / \partial Z_{1}\right)}{(\alpha \hat{y}-1)}+\alpha A_{11},
$$


and the match in $(3.14 \mathrm{c})$ therefore yields the relation

$$
\alpha A_{11}-\frac{i \beta}{\alpha}\left(\frac{\beta \partial P_{01}}{\partial X_{1}}-\frac{\alpha \partial P_{01}}{\partial Z_{1}}\right)=\left(\alpha^{2}+\beta^{2}\right)\left(P_{11}-\frac{i \partial P_{01}}{\partial T_{1}}\right)-i\left(\frac{\alpha \partial P_{01}}{\partial X_{1}}+\frac{\beta \partial P_{01}}{\partial Z_{1}}\right)
$$

Fifth, and allowing for the mean-flow corrections $\hat{U}_{1 M}, \hat{W}_{1 M}$ which are discussed in Appendix B (see also [29]), we find from (3.14d, e, f) the pressuredisplacement relation

$$
\begin{aligned}
&-\frac{i \beta}{\alpha}(\left.\frac{\beta \partial P_{01}}{\partial X_{2}}-\frac{\alpha \partial P_{01}}{\partial Z_{2}}\right)+\alpha A_{21}+i \int_{0}^{\infty} Q d \hat{y}=-\frac{P_{01}}{\alpha m}+\frac{P_{21}}{\alpha^{2}}-\frac{i}{\alpha^{2}} \frac{\partial P_{01}}{\partial T_{2}} \\
&- \frac{\beta}{\alpha}\left(\beta \frac{\partial^{2} P_{01}}{\partial X_{1} \partial T_{1}}-\alpha \frac{\partial^{2} P_{01}}{\partial Z_{1} \partial T_{1}}\right)-i \alpha \frac{\partial A_{11}}{\partial T_{1}}+\frac{P_{01}}{\alpha^{2}} \hat{Q}_{1 M}(0) \\
&-i\left(\alpha \frac{\partial P_{01}}{\partial X_{2}}+\beta \frac{\partial P_{01}}{\partial Z_{2}}\right)-i\left(\alpha \frac{\partial P_{11}}{\partial X_{1}}+\beta \frac{\partial P_{11}}{\partial Z_{1}}\right)+\frac{P_{01}^{*}}{\alpha^{4}}\left(P_{12}+\frac{P_{01}^{2}}{2 \alpha^{2}}\right),
\end{aligned}
$$

where

$$
\begin{gathered}
Q \equiv-\left\{\frac{\partial^{2} \hat{Q}_{11}}{\partial T_{1} \partial \hat{y}}+\frac{\partial \hat{Q}_{11}}{\partial X_{1}}+\frac{\hat{y} \partial^{2} \hat{Q}_{11}}{\partial X_{1} \partial \hat{y}}-\alpha\left(\frac{\partial \hat{U}_{11}}{\partial X_{1}}+\frac{\partial \hat{W}_{11}}{\partial Z_{1}}\right)\right. \\
\left.-i\left(\alpha^{2}+\beta^{2}\right) \hat{y} P_{01} \frac{\partial^{2} \hat{Q}_{1 M}}{\partial \hat{y}^{2}}\right\} /(\alpha \hat{y}-1) .
\end{gathered}
$$

The right-hand side of ( $3.18 \mathrm{a})$ is specified using (3.10b) above, which is equivalent to setting $\hat{y}=0$ in the momentum equations for $\hat{U}_{21}, \hat{W}_{21}$ derived from Appendix A, apart from the buffer effect mentioned earlier.

The four pressure-displacement relations obtained so far, (3.15b), (3.16d), (3.17b), (3.18a), hold independently of the particular external-flow law in (1.1) and so they have quite general application to a range of high-Reynolds-number flows. In the present context the above four relations are to be coupled with (1.1), in the next Sub-Section 3.3, and this then yields the amplitude equation presented in Sub-Section 3.4. Beforehand, however, it should be remarked that in the outer region II there is a critical layer, albeit a passive one. For all the individual velocity solutions (3.15a), (3.16a), and so on, for $U$ 's, $W$ 's, are algebraically singular at the critical level $\hat{y}=\alpha^{-1}$ where the streamwise wavespeed and the basic fluid speed coincide. The critical layer surrounding $\hat{y}=$ $\alpha^{-1}$ is predominantly nonlinear but its global effects are passive, leaving the above singular behaviour unaltered on either side of the critical layer. The critical layer, like most such layers, can only affect logarithmic singularities and there are no such singularities in the present motion since it starts as a uniform parallel shear flow in the lower deck. A study of nonparallel basic local motions (such as breakaway separating motion and flow over humps), where, by contrast, logarithmic singularities do occur and so activate the critical layer, is given in [16]. 


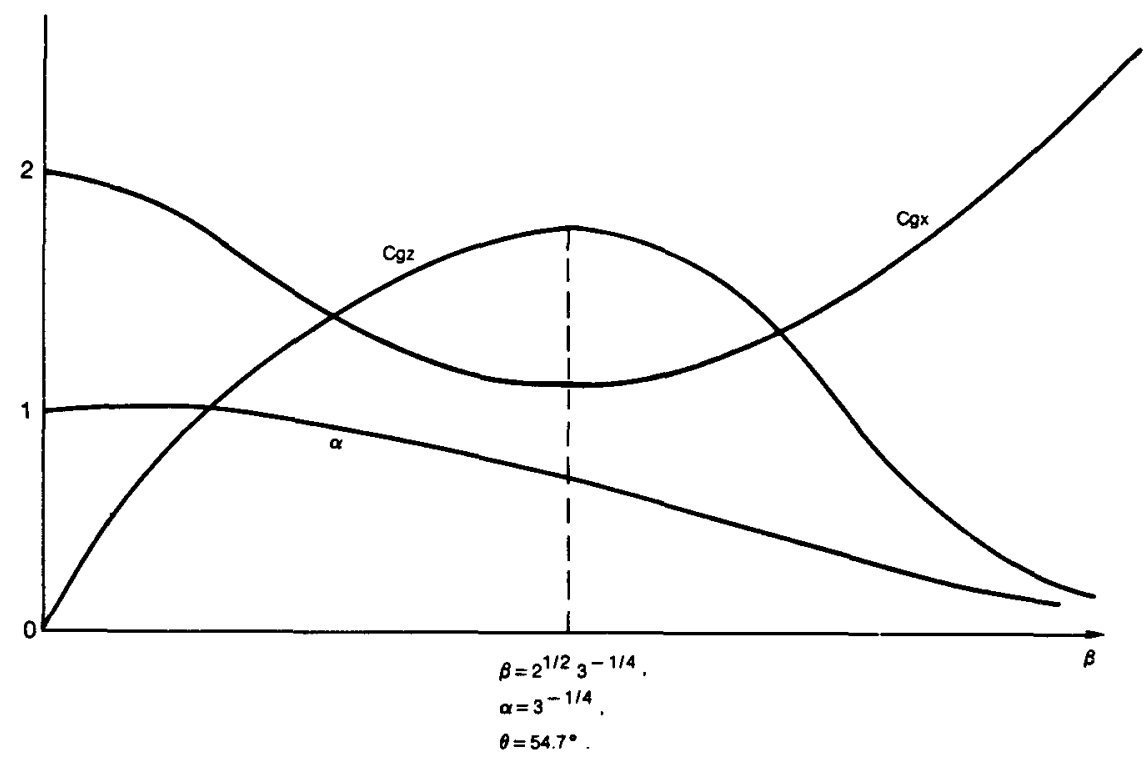

Figure 3. Sketch of the variation of the streamwise wavenumber $\alpha$ and the group velocities $c_{g X}, c_{g z}$, versus the cross-stream wavenumber $\beta$, from Sub-Section 3.3. The critical angle $\theta=\theta_{\text {crit }}$ in (3.28) is shown.

Sub-Section 3.3. The Wavenumbers and Compatibility Relations. We apply now the subsonic pressure-displacement law of (1.1). With the expansions for $P, A$ given in (3.2), followed by the expressions (3.4) for the $P_{n}$ 's and the corresponding ones for the $A_{n}$ 's, the law (1.1) yields successively

$$
\begin{gathered}
\left(\alpha^{2}+\beta^{2}\right)^{1 / 2} P_{01}=\alpha^{2} A_{01}, \\
\left(\alpha^{2}+\beta^{2}\right)^{1 / 2} P_{12}=2 \alpha^{2} A_{12}, \\
\left(\alpha^{2}+\beta^{2}\right)^{1 / 2} P_{11}-i\left(\alpha^{2}+\beta^{2}\right)^{-1 / 2}\left(\alpha \frac{\partial P_{01}}{\partial X_{1}}+\beta \frac{\partial P_{01}}{\partial Z_{1}}\right)=\alpha^{2} A_{11}-2 i \alpha \frac{\partial A_{01}}{\partial X_{1}}, \\
\left(\alpha^{2}+\beta^{2}\right)^{1 / 2} P_{21}-\pi_{21}=\alpha^{2} A_{21}-\frac{2 i \alpha \partial A_{11}}{\partial X_{1}}-\left(\frac{2 i \alpha \partial}{\partial X_{2}}+\frac{\partial^{2}}{\partial X_{1}^{2}}\right) A_{01},
\end{gathered}
$$

after some manipulation. Here

$$
\begin{aligned}
2\left(\alpha^{2}+\beta^{2}\right)^{1 / 2} \pi_{21}= & -\frac{\left(\alpha \partial / \partial X_{1}+\beta \partial / \partial Z_{1}\right)^{2} P_{01}}{\left(\alpha^{2}+\beta^{2}\right)}+2 i\left(\alpha \partial / \partial X_{1}+\beta \partial / \partial Z_{1}\right) P_{11} \\
& +2 i\left(\alpha \partial / \partial X_{2}+\beta \partial / \partial Z_{2}\right) P_{01}+\left(\partial^{2} / \partial X_{1}^{2}+\partial^{2} / \partial Z_{1}^{2}\right) P_{01}
\end{aligned}
$$

The four relations (3.19) are combined below with the four relations of the previous sub-section, to yield compatibility conditions for the nonlinear disturbance.

From (3.19a) and $(3.15 b)$, we obtain the wavenumber relation

$$
\alpha^{2}\left(\alpha^{2}+\beta^{2}\right)=1 \text {, }
$$

fixing $\alpha$ for a given cross-stream wavenumber $\beta$ : Fig. 3 . This is consistent with the eigenrelation (1.3) for high frequencies. 
From (3.19b) coupled with (3.16d), the second-harmonic pressure contribution,

$$
P_{12}=-\left(\alpha^{2}+\beta^{2}\right) P_{01}^{2}
$$

is determined.

From (3.19c) with (3.17b), the governing equation

$$
\left(2 \alpha^{2}+\beta^{2}\right) \frac{\partial P_{01}}{\partial X_{1}}+\frac{\alpha \beta \partial P_{01}}{\partial Z_{1}}+\alpha\left(\alpha^{2}+\beta^{2}\right) \frac{\partial P_{01}}{\partial T_{1}}=0,
$$

is then obtained, which describes the travel of the pressure-disturbance amplitude in a moving frame. The disturbance is a function of $\left(X_{1}-c_{g X} T_{1}\right)$, $\left(Z_{1}-c_{g z} T_{1}\right)$, travelling in the $X_{1}-Z_{1}$ plane with a three-dimensional group velocity whose components are $c_{g X}=\left(2 \alpha^{2}+\beta^{2}\right) /\left(\alpha^{2}+\beta^{2}\right) \alpha, c_{g Z}=\beta /\left(\alpha^{2}+\beta^{2}\right)$ respectively, shown in Fig. 3. This too agrees with the high-frequency limit of the dispersion relation (1.3), as well as with the earlier planar-flow theory.

Finally, from (3.19d), (3.18), the nonlinear equation

$$
\begin{aligned}
& \beta\left(\beta \partial P_{01} / \partial X_{2}-\alpha \partial P_{01} / \partial Z_{2}\right) / \alpha-\int_{0}^{\infty} Q d \hat{y}-2\left(\partial A_{11} / \partial X_{1}+\partial A_{01} / \partial X_{2}\right) \\
& \quad+i\left(\partial^{2} A_{01} / \partial X_{1}^{2}-\pi_{21}\right) / \alpha \\
& =\left(\alpha^{2}+\beta^{2}\right)\left(\partial P_{11} / \partial T_{1}-i \partial^{2} P_{01} / \partial T_{1}^{2}\right)-i\left(\alpha \partial^{2} / \partial X_{1} \partial T_{1}+\beta \partial^{2} / \partial Z_{1} \partial T_{1}\right) P_{01} \\
& \quad+\left(\alpha^{2}+\beta^{2}\right) \partial P_{01} / \partial T_{2}-\frac{i}{2 \alpha^{6}}\left|P_{01}\right|^{2} P_{01}-i \alpha\left(\alpha^{2}+\beta^{2}\right) P_{01} / m \\
& \quad+\left(\alpha \partial / \partial X_{2}+\beta \partial / \partial Z_{2}\right) P_{01}+\left(\alpha \partial / \partial X_{1}+\beta \partial / \partial Z_{1}\right) P_{11}+\frac{i P_{01}}{\alpha^{2}} \hat{Q}_{1 M}(0)
\end{aligned}
$$

controlling $P_{01}$ is derived. Here the functions $\pi_{21}, A_{01}, Q$ and the dependence of $A_{11}$ on $P_{11}$, are given in the previous sub-sections while details of the meanflow equations at higher order that control $\hat{Q}_{1 M}$ are given in Appendix B. Hence, assuming (without loss of generality) that $P_{11}$ also travels with the group velocity, as in (3.22), we have from (3.23)

$$
\begin{aligned}
& -\frac{i}{\alpha}\left\{\left(2 \alpha^{2}+\beta^{2}\right) \partial P_{01} / \partial X_{2}+\alpha \beta \partial P_{01} / \partial Z_{2}+\alpha\left(\alpha^{2}+\beta^{2}\right) \partial P_{01} / \partial T_{2}\right\} \\
& \quad-\frac{\alpha}{m}\left(\alpha^{2}+\beta^{2}\right) P_{01}-\frac{1}{2 \alpha^{6}} P_{01}\left|P_{01}\right|^{2} \\
& =\frac{1}{2}\left(5+\alpha^{4}\right) \partial^{2} P_{01} / \partial X_{1}^{2}+\frac{1}{2}\left(1+\alpha^{2} \beta^{2}\right) \partial^{2} P_{01} / \partial Z_{1}^{2} \\
& \quad+\frac{\beta}{\alpha}\left(2+\alpha^{4}\right) \frac{\partial^{2} P_{01}}{\partial X_{1} \partial Z_{1}}+\frac{1}{\alpha}\left(3 \alpha^{2}+\beta^{2}\right) \frac{\partial^{2} P_{01}}{\partial X_{1} \partial T_{1}}+2 \beta \frac{\partial^{2} P_{01}}{\partial Z_{1} \partial T_{1}} \\
& \quad+\left(\alpha^{2}+\beta^{2}\right) \frac{\partial^{2} P_{01}}{\partial T_{1}^{2}}-\alpha^{-2} P_{01}\left\{\hat{Q}_{1 M}(0)+\int_{0}^{\infty}(\alpha \hat{y}-1)^{-2} \hat{Q}_{1 M \hat{y}} d \hat{y}\right\} .
\end{aligned}
$$


But the time derivatives $\partial / \partial T_{1}$ can be eliminated in favour of spatial derivatives $\partial / \partial X_{1}, \partial / \partial Z_{1}$ in view of the moving-frame property $(3.22)$, and likewise the combination of first derivatives with respect to $X_{2}, Z_{2}, T_{2}$ acts as a single time derivative in a frame moving with the group velocity. The resulting amplitude equation for $P_{01}$ is given in the following sub-section.

Sub-Section 3.4. The Amplitude Equation, and the Critical Angle. From (3.24), after some further working, the nonlinear evolution equation for the unknown pressure function $P_{01}$ is found to be

$$
\begin{array}{r}
2 \frac{\partial P_{01}}{\partial T_{2}}-i\left[\alpha^{2}\left(3-\alpha^{4}\right) \frac{\partial^{2} P_{01}}{\partial X_{1}^{2}}+2 \alpha \beta\left(1-\alpha^{4}\right) \frac{\partial^{2} P_{01}}{\partial X_{1} \partial Z_{1}}+\alpha^{6} \frac{\partial^{2} P_{01}}{\partial Z_{1}^{2}}\right] \\
=2 i \alpha^{3}\left(\alpha^{2}+\beta^{2}\right) \exp (-3 \pi i / 4) P_{01}+i \alpha^{-4} P_{01}\left|P_{01}\right|^{2} \\
-2 i P_{01}\left\{\hat{Q}_{1 M}(0)+\int_{0}^{\infty}(\alpha \hat{y}-1)^{-2} \hat{Q}_{1 M \hat{y}} d \hat{y}\right\}
\end{array}
$$

with,

$$
\begin{gathered}
\hat{Q}_{1 M \hat{y}}=\frac{\left(\beta \partial / \partial X_{1}-\alpha \partial / \partial Z_{1}\right)}{\alpha^{2}(\alpha \hat{y}-1)^{2}} \partial_{z_{1}} \int_{0}^{\infty} \sigma\left|P_{01}\right|^{2}\left\{X_{1}-\sigma\left(\hat{y}-c_{1}\right), Z_{1}+\sigma c_{2}\right\} d \sigma \\
\hat{Q}_{1 M}(0)=-\alpha^{-2}\left(\alpha \partial / \partial X_{1}+\beta \partial / \partial Z_{1}\right) \int_{0}^{\infty}\left|P_{01}\right|^{2}\left\{X_{1}+\sigma c_{1}, Z_{1}+\sigma c_{2}\right\} d \sigma
\end{gathered}
$$

where $c_{1}=\alpha\left(2 \alpha^{2}+\beta^{2}\right), c_{2}=\alpha^{2} \beta$ are constants. Details of the mean-flow equations leading to $(3.25 \mathrm{~b}, \mathrm{c})$ are presented in Appendix B. In effect $(3.25 \mathrm{a}-\mathrm{c})$ give $P_{01}$ as a function of the moving spatial coordinates $X_{1}, Z_{1}$ as time $T_{2}$ progresses. Here, for a given $\beta$, the value of $\alpha$ is determined from (3.20). All the linear terms in (3.25) can be shown to be in agreement with the highfrequency limit of the dispersion relation, (1.3), in fact, although the derivation via Sub-Sections 3.1-3.3 demonstrates much more clearly the structure of the flow and obtains the nonlinear effects proportional to $P_{01}\left|P_{01}\right|^{2}, P_{01} \hat{Q}_{1 M}$. The linear-growth term proportional to $P_{01}$, in particular, comes from viscous effects, specifically from the Stokes-layer displacement, whereas viscous forces do not appear to influence the nonlinear terms in (3.25). Other relevant aspects at this juncture, including the range of validity, are much as in the planar-flow case in $[8,9]$ with which (3.25) agrees when $\beta$ is set equal to zero and $\alpha \rightarrow 1$, from (3.20).

For the rest of this section and in Sections 4, 5 we address the interesting special case of negligible mean-flow correction $\left(\hat{Q}_{1 M}\right)$, which is justified later in Section 6 where more general cases are considered. So the equation (3.25a) can now be put into canonical form by means of the coordinate transformation: 


$$
\begin{aligned}
& \xi=\frac{2^{1 / 4} X_{1}}{\left(3-\alpha^{4}\right)^{1 / 2} \alpha^{1 / 2}}, \\
& \eta=\frac{2^{1 / 4} \alpha^{1 / 2}}{\left|3 \alpha^{4}-1\right|^{1 / 2}}\left\{\frac{\beta\left(1-\alpha^{4}\right) X_{1}}{\left(3-\alpha^{4}\right)^{1 / 2}}-\alpha\left(3-\alpha^{4}\right)^{1 / 2} Z_{1}\right\},
\end{aligned}
$$

where we note that $0<\alpha<1$ from (3.20); and by defining a normalized pressure $\hat{P}$ and time $\hat{T}$ by

$$
P_{01}=\frac{\alpha^{2}}{5^{1 / 2}} \exp \left(\frac{-i \alpha T_{2}}{2^{1 / 2}}\right) \hat{P}, \quad T_{2}=\frac{2^{1 / 2}}{\alpha} \hat{T}
$$

This changes (3.25) to the governing equation

$$
\frac{\partial \hat{P}}{\partial \hat{T}}-i\left(\frac{\partial^{2} \hat{P}}{\partial \xi^{2}} \pm \frac{\partial^{2} \hat{P}}{\partial \eta^{2}}\right)=\hat{P}-i \hat{P}|\hat{P}|^{2},
$$

for $\hat{P}(\xi, \eta, \hat{T})$, subject to prescribed initial conditions at time $\hat{T}=0$ and appropriate spatial constraints. The \pm signs in (3.27) apply respectively for $\alpha \gtrless$ $3^{-1 / 4}$, which correspond to cross-stream eavenumbers $\beta \lessgtr 2^{1 / 2} 3^{-1 / 4}$, from (3.20). So a crossover, or critical wave angle, occurs at the value $\beta / \alpha=\sqrt{2}$. The spatial character of the governing equation therefore changes from elliptic (for $\theta<\theta_{\text {crit }}$ ) to hyperbolic (for $\theta>\theta_{\text {crit }}$ ) as the wave angle $\theta$ passes through the critical value

$$
\theta_{\text {crit }}=\tan ^{-1}(\sqrt{2})\left[\doteqdot 54 \cdot 7^{\circ}\right] \text {. }
$$

For $\theta<\theta_{\text {crit }}$ the + sign holds in (3.27), while for $\theta>\theta_{\text {crit }}$ the - sign holds. This change in sign has significant impact, as the next sections show.

Numerical solutions are still required generally for the full threedimensional amplitude equation (3.27) and these have yet to be obtained. They should prove to be of much interest, according to the predictions found below for the simpler case of oblique three-dimensional waves and the contrasting predictions of the two-dimensional theory in [9] (see also the next section). Added to this is the related issue of the crossover from an elliptic to a hyperbolic nature at the critical angle in (3.28). The crossover is indeed important when oblique waves are considered, in the following section.

\$4. Three-dimensional oblique waves. Here and in Section 5 we continue with the special case of negligible mean-flow influence. For an oblique wave sent through the flow system, we seek the separation-of-variables solution

$$
\hat{P}=e^{\mathrm{i} n \xi} \vec{e}^{\mathrm{i} n^{2} \hat{T}} Q(\eta, \hat{T}) .
$$

Here $n$ is a real constant allowing for extra $\xi$-waviness, but this does not affect the outcome. Now (3.27) reduces to the nonlinear amplitude equation

$$
\frac{\partial Q}{\partial \hat{T}} \mp i \frac{\partial^{2} Q}{\partial \eta^{2}}=Q-i Q|Q|^{2},
$$

for $Q(\eta, \hat{T})$, subject to a prescribed initial distribution $Q(\eta, 0)$ and the boundary conditions of periodicity in $\eta$ or $Q \rightarrow 0$ as $\eta \rightarrow \pm \infty$. The solution properties 
depend on the $\mp$ signs in (4.2干), applying for $\theta$ below and above $\theta_{\text {crit }}$ respectively.

When $\theta<\theta_{\text {crit }}$ and the - sign holds, (4.2-) is effectively the same as the two-dimensional case of [9] where $\beta \rightarrow 0, \alpha \rightarrow 1$. See also the computations and further comments below.

For the $+\operatorname{sign}$, corresponding to oblique waves at angles exceeding $\theta_{\text {crit }}$ in (3.28), computations were performed as described subsequently. First, however, we show the existence of a form of sideband instability. This is most easily seen in a perturbation about the uniform-state solution,

$$
Q=Q_{0}(\hat{T})=A_{0} \exp \left\{\hat{T}-i A_{0}^{2} \exp (2 \hat{T}) / 2\right\}
$$

[ $A_{0}$ is a positive constant], of $(4.2+)$, in the form

$$
Q=Q_{0}(\hat{T})+\varepsilon Q_{1}(\eta, \hat{T})+O\left(\varepsilon^{2}\right),
$$

where $\varepsilon$ is small. Here, from $(4.2+)$ at $O(\varepsilon)$, the perturbation in modulus $R_{1} \equiv|Q|-\left|Q_{0}\right|$ satisfies

$$
\frac{\partial^{4} R_{1}}{\partial \eta^{4}}+2 A_{0}^{2} e^{2 \hat{T}} \frac{\partial^{2} R_{1}}{\partial \eta^{2}}+\left(\frac{\partial}{\partial \hat{T}}-1\right)^{2} R_{1}=0
$$

So, if $R_{1}=R_{1}^{*}(\hat{T}) \exp \left(i \alpha^{*} \eta\right)$, which is equivalent to the taking of a Fourier transform in $\eta, s^{-1} R_{1}^{*}$ is controlled by the Besel equation

$$
\left[s^{2} \frac{\partial^{2}}{\partial s^{2}}+s \frac{\partial}{\partial s}+\left(\alpha^{* 4}-2 A_{0}^{2} \alpha^{* 2} s^{2}\right)\right]\left(\frac{R_{1}^{*}}{s}\right)=0
$$

in terms of $s \equiv \exp (\hat{T})$, for any perturbation wavenumber $\alpha^{*}$. From (4.3c), therefore, the perturbation amplitude $R_{1}^{*}$ grows primarily like $\exp \left(\sqrt{2} A_{0} \alpha^{*}\right.$ s) for large $\alpha^{*}$ s, i.e. like $\exp \left\{\sqrt{2} A_{0} \alpha^{*} \exp (\hat{T})\right\}$ at large $\alpha^{*} \exp (\hat{T})$. The growth of the sideband perturbation, then, is very fast, proportional to exp (exp (time)) schematically, and moreover it is faster for the shortest waves (large $a^{*}$ ) and increases as the uniform-state amplitude $A_{0}$ increases. In contrast, the planar and $\theta<\theta_{\text {crit }}$ cases (4.2-) yield no sideband instability. Hence the integration of $(4.2+)$ is especially intriguing. Further support for this comes from the property that the double derivative in $(4.2+)$ tends to yield spatial focussing as opposed to the spreading which holds in the planar case.

We concentrate therefore on the case $(4.2+)$, associated with

$$
\theta>\theta_{\text {crit }} \text {. }
$$

Numerical solutions were obtained by use of an explicit predictor-corrector finite-difference scheme, of nominal second-order accuracy in time and space. For each small time step from $\hat{T}$ to $\hat{T}+\Delta \hat{T}$, the explicit predictor $Q$-pred from $(4.2+)$ is

$$
Q-\operatorname{pred}_{k}=\bar{Q}_{k}+\Delta \hat{T}\left\{\bar{Q}_{k}-i\left[\frac{\bar{Q}_{k+1}-2 \bar{Q}_{k}+\bar{Q}_{k-1}}{(\Delta \eta)^{2}}\right]-i \bar{Q}_{k}\left|\bar{Q}_{k}\right|^{2}\right\}
$$


giving $Q$-pred for $_{k} \leqslant k \leqslant K-1$, followed by the corrector $Q$-corr ${ }_{k}$ in the form $Q-\operatorname{corr}_{k}=\frac{1}{2}\left(Q-\operatorname{pred}_{k}+\bar{Q}_{k}\right)$

$$
\begin{array}{r}
+\frac{1}{2} \Delta \hat{T}\left\{Q-\operatorname{pred}_{k}-i\left[\frac{Q-\operatorname{pred}_{k+1}-2 Q-\operatorname{pred}_{k}+Q-\operatorname{pred}_{k-1}}{(\Delta \eta)^{2}}\right]\right. \\
\left.-i Q-\operatorname{pred}_{k}\left|Q-\operatorname{pred}_{k}\right|^{2}\right\},
\end{array}
$$

again for $2 \leqslant k \leqslant K-1$. Here the subscript $k$ refers to evaluation at the typical gridpoint $\eta=\eta_{k}=\eta_{1}+(k-1) \Delta \eta$, where $\Delta \eta$ is the small $\eta$-step and $\eta=\eta_{1}, \eta_{K}$ are the end points of the integration range, while the overbar denotes the known values at the previous time level $\hat{T}$. After (4.5b) is applied $\bar{Q}$ is then replaced by $Q$-corr for the next time step. The end values $Q_{1}, Q_{K}$ are kept fixed at zero throughout. Tests applied in [47], to check on the effects of the grid spacings, the time step, and the large end values $\eta_{1}$ (negative), $\eta_{K}$ (positive) indicate that the numerical accuracy is approximately graphical for the discretizations taken.

Numerical results are presented here for two examples of nonsymmetric initial conditions. The first has an initial distribution of a one-humped form,

$$
Q(\eta, 0)=\frac{\exp \left(3 \eta-\left(\eta^{2} / 9\right)\right)}{[1+\exp (3 \eta)]}
$$

and the results are shown in Fig. 4, while Fig. 5 shows the solution obtained for the two-humped starting condition

$$
Q(\eta, 0)=\frac{\exp \left(3 \eta-\left(\eta^{2} / 9\right)\right)}{[1+\exp (3 \eta)]}+\exp \left(-\frac{1}{9}(\eta+3)^{2}\right)
$$

Both sets of results show fairly clear signs at early times of the secondary or sideband instability mechanism and focussing described previously. Growing waves start to appear and they continue to amplify even as they become comparable in size with, or larger than, the underlying solutions. Eventually the fastest-growing shorter-scale waves seem to take over in the nonlinear solution, tending towards spike-like behaviour, and a chaotic appearance then develops. Spectra of the solutions at various times $\hat{T}$ are also presented in [47]. Each spectrum there was derived numerically by calculating the values of the coefficients of the Fourier series for $Q$ in the computational interval $\eta_{1}<\eta<\eta_{K}$. These values also were checked by evaluation on a number of grids. The principal feature observed in the results is the pronounced broadening of the spectrum as time increases, in line with the enhancement of the spikes mentioned above.

As a further check on the accuracy of the numerical scheme calculations were also performed for the planar or $\theta<\theta_{\text {crit }}$ case of $(4.2-)$. The results are given in Fig. 6 and are compared with the earlier computations of [9]. The agreement seems satisfactory and backs up the broad spreading effect in the planar-flow solution, as noted in that reference. Also shown is the development of the spectrum of this solution, which verifies the lack of spectrum broadening 


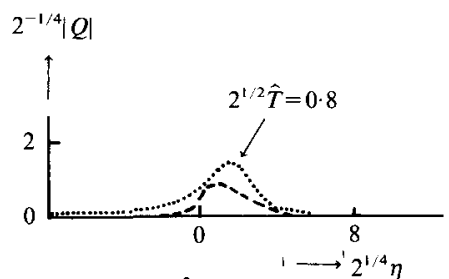

(1) $2^{1 / 2} \hat{T}=0$ and $0 \cdot 8$

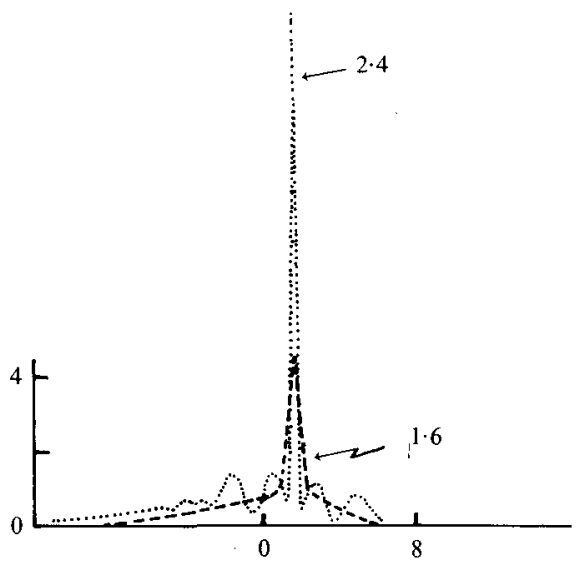

(2) $2^{1 / 2} \hat{T}=1.6$ and $2 \cdot 4$

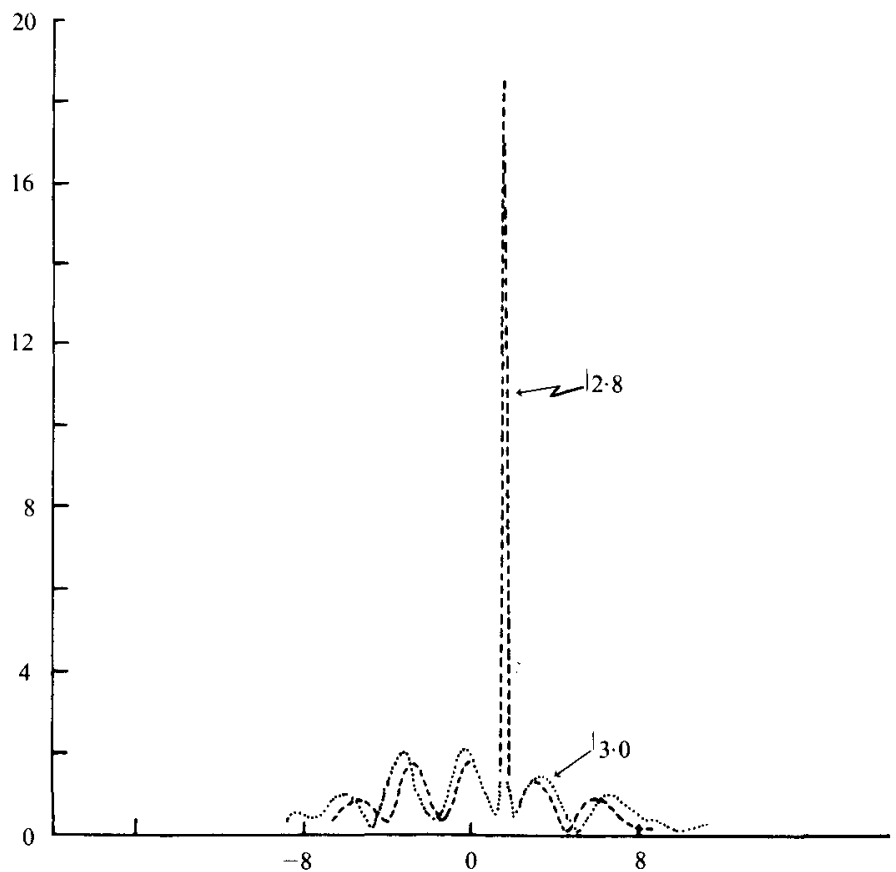

(3) $2^{1 / 2} \hat{T}=2 \cdot 8$ and $3 \cdot 0$

Figure 4. Numerical solutions of $(4.2+)$ for the effective pressure amplitude $|Q|$, given the one-humped starting form (4.6a), from grid $[\Delta \hat{T}, \Delta \eta, K]=[0.0004,0.2,201]$. The spike has been truncated in the case when $2^{1 / 2} \tilde{T}=3 \cdot 0$. See also [47]. 


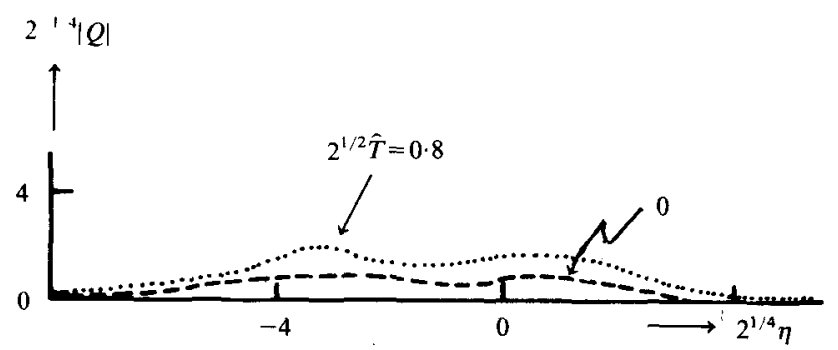

(1) $2^{1 / 2} \hat{T}=0$ and $0 \cdot 8$

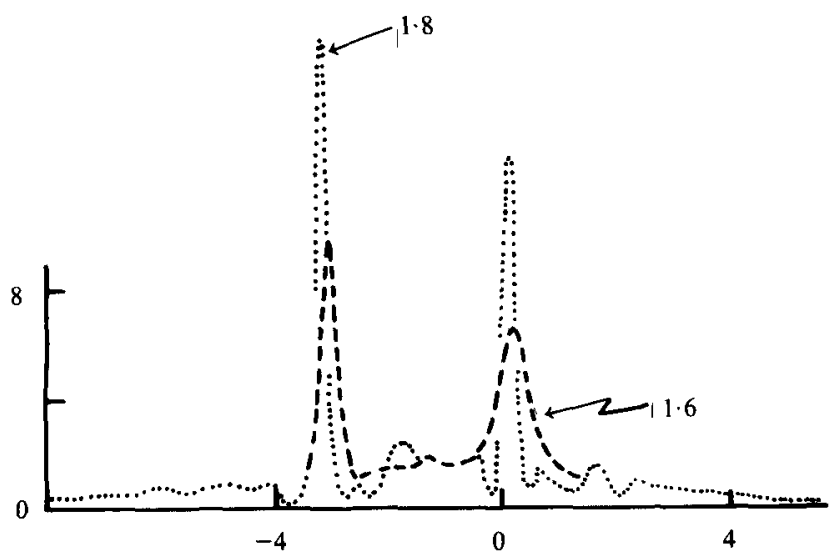

(2) $2^{1 / 2} \hat{T}=1.6$ and 1.8

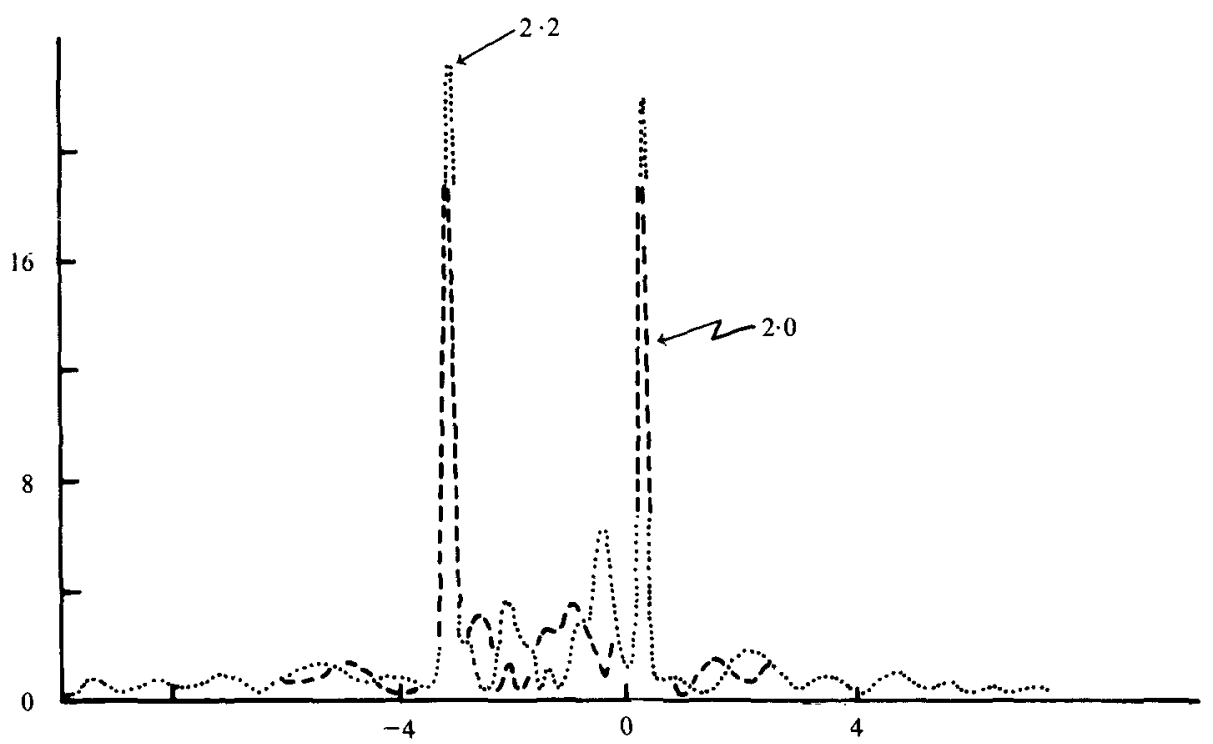

(3) $2^{1 / 2} \hat{T}=2 \cdot 0$ and $2 \cdot 2$

Figure 5. Computations of $(4.2+)$ for the two-humped starting form in (4.6b) showing the effective pressure distribution $|Q|$ at various times $\hat{T}$, for grid $[0.0001,0.05,401]$. 

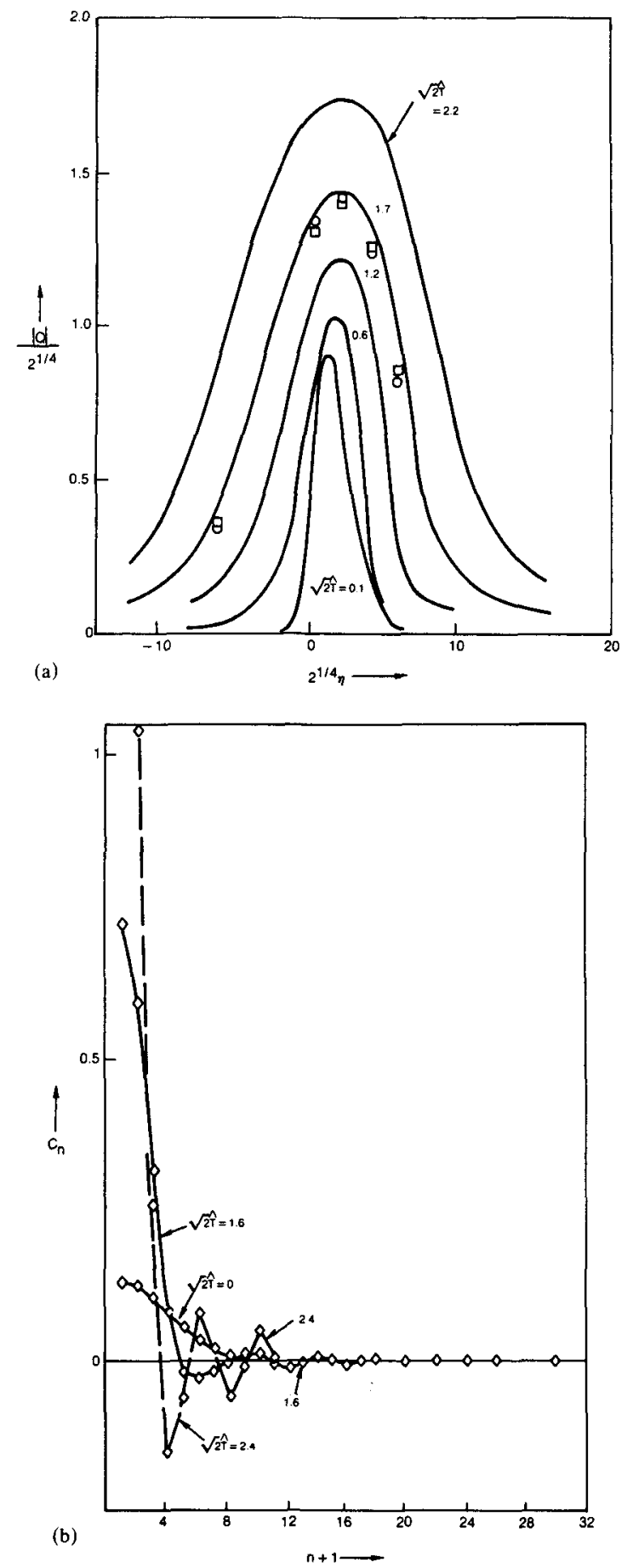

Figure 6. Check calculations of the two-dimensional case (4.2-), or $\theta<\theta_{\text {crit }}$. (a) A comparison of results at $\sqrt{2} \hat{T}=1.6$ obtained from the present method [O] and from [9] ( $\square$, using interpolation of the solutions _- at other times). (b) The amplitude's spectral cosine components $C_{n}$, versus $n$, at times $\sqrt{2} \hat{T}=0,1 \cdot 6,2 \cdot 4$. The initial distribution here is (4.6a). Cf. the properties in Figs. 4, 5 and [47]. 
for (4.2-), apart from an oscillatory tail, as described in [9]. The ultimate form of the solution here, at larger times $\hat{T}$, has a massive elliptical shape, with the amplitude $|Q|$ growing as $\exp (2 T / 3)$ and the typical $\eta$-range also spreading out exponentially, proportionally to $\exp (2 \hat{T} / 3)$ : see last reference. This applies also for all oblique waves with $\theta<\theta_{\text {crit }}$.

Significantly different features arise in the new case $(4.2+)$, then, as compared with the previously studied case (4.2-). These prompt the investigation in the following section of the ultimate form of the nonlinear solution for $(4.2+)$, when $(4.4)$ holds.

§5. Large-time/far-downstream behaviour of the three-dimensional oblique waves. The computations above point to the pressure amplitude $|Q|$ becoming increasingly large and the characteristic spatial scale $\eta$ shortening as time $T$ increases, in the case $(4.2+)$. An associated limiting description for large times is considered below, this corresponding to a far-downstream form because of the movement in (3.22). The form applies for periodic or non-periodic motions.

The equation $(4.2+)$ is recast in terms of the amplitude and phase, $Q=$ $R \exp (i \varphi)$, where $R, \varphi$ satisfy

$$
\begin{array}{r}
-R \frac{\partial \varphi}{\partial \hat{T}}-\frac{\partial^{2} R}{\partial \eta^{2}}+R\left(\frac{\partial \varphi}{\partial \eta}\right)^{2}=R^{3} \\
-R+\frac{\partial R}{\partial \hat{T}}-2 \frac{\partial R}{\partial \eta} \frac{\partial \varphi}{\partial \eta}-R \frac{\partial^{2} \varphi}{\partial \eta^{2}}=0 .
\end{array}
$$

There is from (5.1b) an integral constraint

$$
\int_{-\infty}^{\infty} R^{2}(\eta, \hat{T}) d \eta=e^{2 \hat{T}} \int_{-\infty}^{\infty} R^{2}(\eta, 0) d \eta,
$$

of exponential growth away from the initial conditions: the constraint (5.2) agrees reasonably well with the calculations. Quite a number of possible limiting descriptions for $(4.2+)$, i.e., $(5.1 \mathrm{a}, \mathrm{b})$, suggest themselves but, cutting matters short, we find that an account that seems appropriate as well as selfconsistent has $R=|Q|$ being very large, of order exp $(2 \hat{T})$, within a typical tiny interval $\left(\eta-\eta_{m}\right)$ of order $\exp (-2 \hat{T})$ in the neighbourhood of a position $\eta=$ $\eta_{m}$. The integral on the left-hand side of (5.2) is then comparable with the right-hand side. The analysis suggests the existence of a number of such tiny intervals. In each one,

$R=e^{2 \hat{T}} \bar{R}(\bar{\eta})+\ldots, \quad \varphi=-K_{m} e^{4 \hat{T}}+e^{-4 \hat{T}} \bar{\varphi}(\bar{\eta})+\ldots, \quad \eta-\eta_{m}=e^{-2 \hat{T}} \bar{\eta}$,

predominantly, for large $\hat{T}$, with $K_{m}$ being an unknown constant. So $(5.1 \mathrm{a}, \mathrm{b})$ reduce to the ordinary differential equations

$$
\begin{gathered}
\bar{R}^{\prime \prime}=4 K_{m} \bar{R}-\bar{R}^{3}, \\
\bar{R}+2 \bar{\eta} \bar{R}^{\prime}=2 \bar{R}^{\prime} \overline{\varphi^{\prime}}+\bar{R} \overline{\varphi^{\prime \prime}},
\end{gathered}
$$




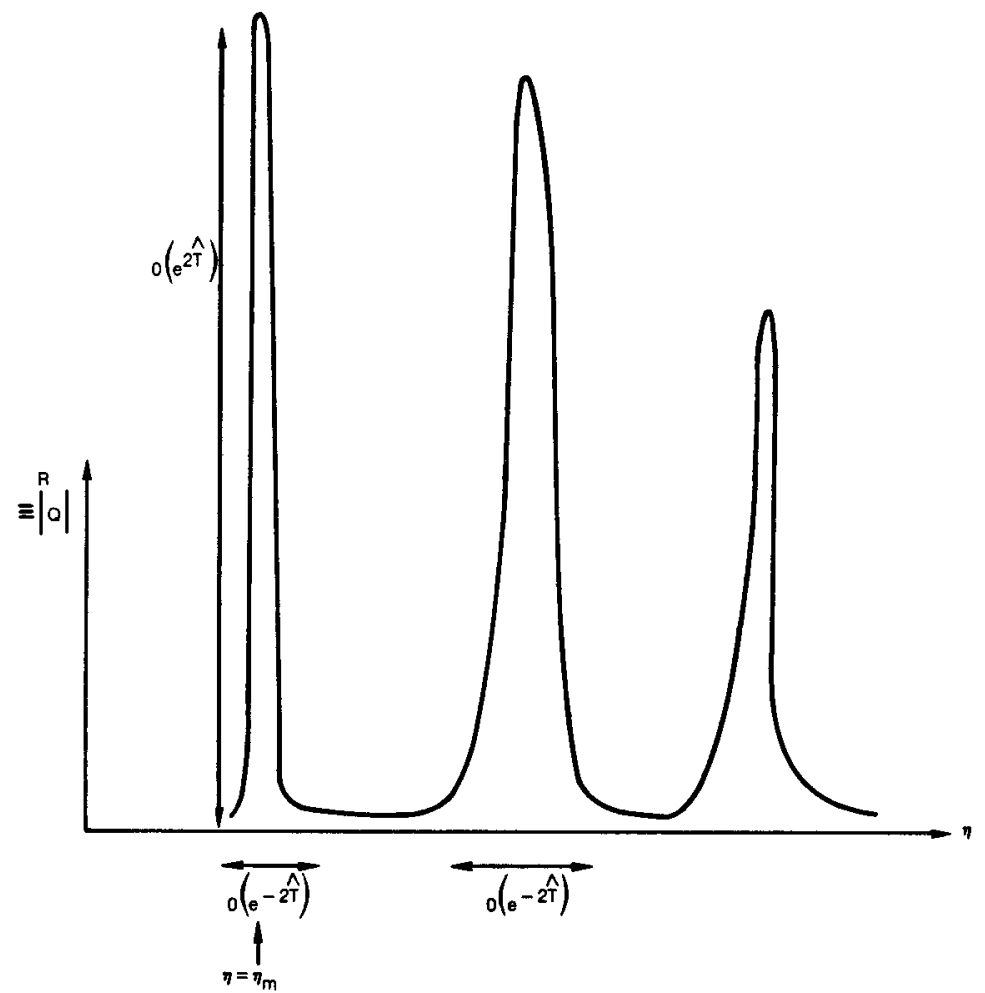

Figure 7. Schematic diagram of the "spikes" structure at large times or far downstream, from Section 5 , for the case $\theta>\theta_{\text {crit }}$.

for $\bar{R}, \bar{\varphi}$. Here the nonlinear equation (5.4a) integrates once to yield $\bar{R}^{2}$ in terms of $\bar{R}$, and since (5.2) requires $\bar{R}( \pm \infty)=0$ it follows that $\bar{R}$ has a soliton sech-form,

$$
\bar{R}=2^{3 / 2} K_{m}^{1 / 2} \operatorname{sech}\left(2 K_{m}^{1 / 2} \bar{\eta}\right)
$$

subject to an origin shift in $\bar{\eta}$, and with $K_{m}$ assumed positive. The solution of (5.4b) well-behaved at infinity is then simply

$$
\vec{\varphi}=\frac{1}{2} \bar{\eta}^{2}+\text { const. }
$$

So (5.3)-(5.6) appear to provide an acceptable terminal account for $(4.2+)$, or (5.1), locally.

There are several points worth noting about the limiting formation above; see also Fig. 7. First, it describes a single spike but there can be any number of such spikes distributed at quite random positions $\eta_{m}$. The effective strength $K_{m}^{1 / 2}$ and position $\eta_{m}$ of each spike are unknown, as far as the limiting form can determine, but their total strength is prescribed by the starting conditions, that is

$$
\sum_{m} K_{m}^{1 / 2}=\frac{1}{8} \int_{-\infty}^{\infty} R^{2}(\eta, 0) d \eta
$$


from (5.2). Second, although the exponentially thin spikes have exponentially large amplitude, each individual has a classical soliton-like form, (5.5). The role of the unstable growth term $\partial R / \partial \hat{T}$ in $(5.1 \mathrm{~b})$, or $\partial Q / \partial \hat{T}$ in $(4.2+)$, which stems from viscous effects, is of interest here. It has no influence in the internal make-up of each spike, in (5.4)-(5.6); its influence comes instead through the overall control of the integral condition (5.2), which in turn leads directly to the scalings (5.3) and the constraint (5.7). This role is quite unlike what happens in the planar-flow case (or $\theta<\theta_{\text {crit }}$ ) of [9]. The question of whether the number of spikes occurring at large times can be infinite or not is also a matter of concern here, since that can affect the strength of each spike, but we believe that the form (5.3) represents the maximum strength possible: see also the next paragraph. Certainly, the limiting description (5.3)-(5.6) seems to tie in with the computations of Section 4 at increased times* as well as with the somewhat related ones in [5], it being noted that the large-time argument above can be modified readily to the spatially bounded problems of the last-named reference and others.

In addition, each of the fast-growing spikes (5.3)-(5.6) can be shown to be stable in itself (see Appendix C), not suffering the sideband growth referred to earlier. This further reinforces belief in the account (5.3)-(5.6). For large $\bar{\eta}$, $\bar{R}$ decays exponentially, while the algebraic growth of $\bar{\varphi}$ indicates the necessity of a new regime, outside the spike, only at very large distances. Further growing spikes can arise in these quiescent regions between established spikes only until the total integral condition (5.7) is satisfied. Again, a possible travel of the spikes, with $\eta_{m}$ then being relatively slowly dependent on time, appears to have little effect on the terminal properties above.

The main property of the three-dimensional case $(4.2+)$, where $\theta>\theta_{\text {crit }}$, is clearly that the nonlinear amplitude growth (proportional to exp $(2 \hat{T}))$ is much greater than in the planar-flow or $\theta<\theta_{\text {crit }}$ case (which yields growth proportional to $\exp (2 \hat{T}) / 3$ ) and also greatly exceeds the linear-theory growth proportional to $\exp (\hat{T})$. This is due solely to the difference in signs in (4.2 \pm$)$. It may be possible, in fact, to combine the different asymptotes for the two cases to provide a limiting description for the general three-dimensional problem (3.27), but that remains an open question as yet, as does the alternative of a finitetime breakdown. The theoretical significance of the critical angle $\theta_{\text {crit }}$ in (3.28) also has a resemblance with experimental results: see Section 7 below. The more immediate repercussions of the fast growth, for the case $(4.2+)$, are considered in Section 7, after a study of more general cases in the next section.

\$6. More general cases. Generally the mean-flow effect proportional to $\hat{Q}_{1 M}$ in $(3.25 \mathrm{a})$ must be coupled with $P_{01}$ via $(3.25 \mathrm{~b}, \mathrm{c})$. If, by analogy with

* Further, the Fourier transform (in $\eta$ ) of the amplitude predicted by (5.3), (5.5) for large $\hat{T}$ is $2^{1 / 2} \pi \exp \left(-i n \eta_{m} \operatorname{sech}\left[\pi n \exp (-2 \hat{T}) /\left(4 k_{m}^{1 / 2}\right)\right]\right.$, for each spike, where $n$ is the transform variable. A sum of such terms is reasonably consistent with the calculated spectral properties at increased $\hat{T}$ in Figs. 4(c), $5(\mathrm{e})$ of $[47]$. 
Section 4, oblique waves are considered in which the $X_{1}-Z_{1}$ dependence occurs through (say) $\eta_{1} \equiv X_{1}+M Z_{1}$ in essence, then from $(3.25 \mathrm{~b}, \mathrm{c})$

$$
\begin{aligned}
& \hat{Q}_{1 M}(0) \rightarrow \alpha^{-1} \hat{c}^{-1}(\alpha+\beta M)\left|P_{01}\right|^{2}, \\
& \int_{0}^{\infty}(\alpha \hat{y}-1)^{-2} \hat{Q}_{1 M \hat{y}} d \hat{y} \rightarrow \alpha(\beta-\alpha M) M \\
& \quad \times\left\{I\left|P_{01}\right|^{2}+4 \alpha^{-2}(\hat{c}-1)^{-5} \operatorname{sgn}(M) \int_{-\infty}^{\infty} \frac{\left|P_{01}\right|^{2}\left(\eta^{\prime}\right) d \eta^{\prime}}{\left(\eta^{\prime}-\eta_{1}\right)}\right\} .
\end{aligned}
$$

Here the constants $\hat{c} \equiv \alpha\left(c_{1}+M c_{2}\right), I \equiv f_{0}^{\infty}(\alpha y-1)^{-4}(\alpha y-\hat{c})^{-2} d y$. Further details are given in Appendix B. So effectively in normalized terms a CauchyHilbert integral term is added to (3.25a) in general. If, however, $\beta=\alpha M$ for example then the coefficient multiplying that integral is zero and so all the working of Sections 4 and 5 then applies.

Hence equations (4.2干) are replaced by

$$
\frac{\partial Q}{\partial \hat{T}} \mp i \frac{\partial^{2} Q}{\partial \eta^{2}}=Q-i Q|Q|^{2}+i \varepsilon Q \int_{-\infty}^{\infty} \frac{|Q|^{2}(\hat{\eta}, \hat{T}) d \hat{\eta}}{(\eta-\hat{\eta})}
$$

where the constant $\varepsilon$ may be zero or nonzero. Numerical solutions of (6.2) for various nonzero values of $\varepsilon$ were obtained by a spectral method for flows periodic in $\eta$, which are our main concern here. The example shown in Fig. 8(a)-(c) is for large $\varepsilon$, effectively, in the sense that the first nonlinear term in (6.2) is omitted and the second one has $\varepsilon$ equal to unity [cf. Section 4]. The behaviour with increasing time is eventually distinct from that in Section 4, with Fig. 8(a)-(c) showing faster spatial variation in the phase than in the amplitude.

In keeping with Fig. 8(a) (c), it is felt that the account of Section 5 no longer applies if $\varepsilon$ is nonzero. Thus an expansion, for small $\varepsilon$, about (5.5) is found to lead to an inconsistency in the generalized version of (5.4), due to an interplay of oddness and evenness in $\bar{\eta}$. Instead, the large-time response seems to take the form (with $a_{1}$ a positive constant)

$$
R \equiv|Q| \sim a_{1} \exp (\hat{T})+\ldots, \quad \varphi_{\eta} \propto \exp (\hat{T})+\ldots,
$$

which is both consistent with (6.2) for $\hat{T}_{\gg 1}$ and in line with the results in Fig. 8(a)-(c). Typical numerical results for some non-periodic flows are also presented in Fig. 8(d), these being notable for the apparent creation of a strong moving front with increasing time. Orders of magnitude can be estimated for this at large times.

§7. Implications for the next stages in the development of the nonlinear disturbance. Concerning the more interesting special case first, the Sections 3-5 above concern the development of the three-dimensional high-frequency disturbance during the first nonlinear stage 1 . As in the planar case studied 

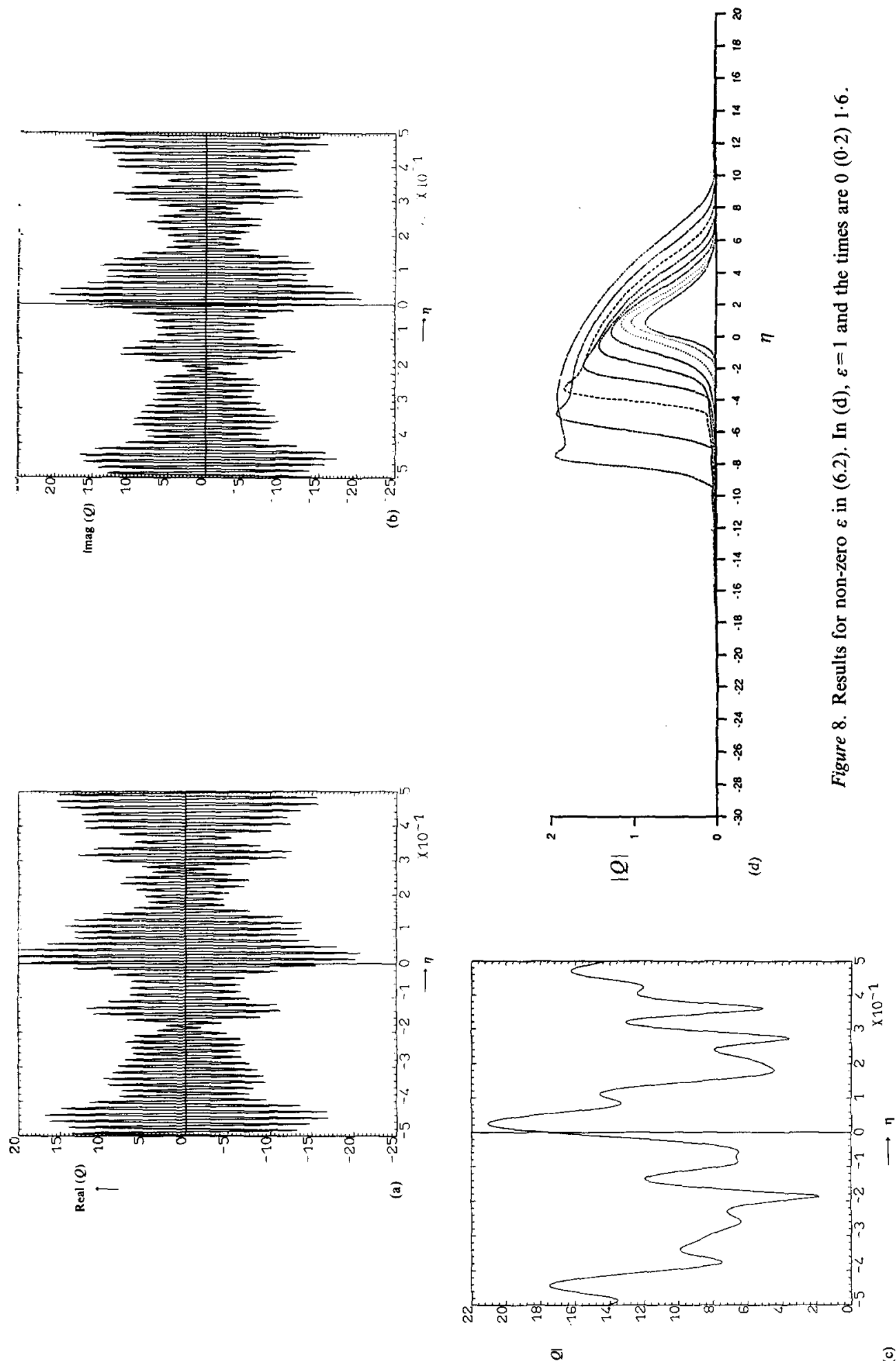
before, a subsequent and more nonlinear stage 2 is reached next as the disturbance travels further downstream. The major difference from the earlier-studied case is simply that the next stage 2 is encountered sooner, for the oblique disturbance of $(4.2+)$.

For from (5.3) compared with (3.5), the phase of the nonlinear disturbance increases temporally at the rate $-\Omega-4 \alpha K_{m} \exp (4 \alpha T)$ predominantly, downstream, in each spike, and so a new balance comes into play downstream when $T$ rises to the order

$$
T \sim \frac{1}{4 \alpha} \ln \Omega .
$$

Similarly, for each spike, the pressure amplitude $|P|$ behaves as $\Omega^{1 / 2} \exp (2 \alpha T)$ downstream, from (3.2a), (5.3), implying that $|P|$ rises to exactly $O(\Omega)$ when (7.1) holds. At that stage the outer region II (Sub-Section 3.2) becomes fully nonlinear, which is the property defining stage 2 . Other estimates also agree generally with the breakdown criterion (7.1). For example, the two major scales in $Z$ are the fastest one $Z_{0} \equiv \beta^{-1} \Omega^{-1 / 2} Z$ and the slower one $Z_{1} \equiv Z$, but $Z_{1}$ itself decreases as $\exp (-2 \alpha T)$ downstream, from (3.26), (5.3), forcing the two scales to coincide exactly at the stage of (7.1). A similar estimate applies to the $X$-scales, for each spike.

More precisely, it appears that the breakdown to stage 2 is reached soonest, spatially and temporally, for a nonlinear growing wave travelling exactly at the critical oblique angle $\theta=\theta_{\text {crit }}$ in (3.28). The group velocities in Sub-Section 3.3 , for instance, suggest that $X_{1}(\approx X)$ scales with $\Omega^{-1 / 2} \alpha^{-2}(\alpha T)$, which with (7.1) tends to imply that the shortest streamwise distance to breakdown occurs for the largest $\alpha$ under which (7.1) continues to hold, i.e., for $\theta=\theta_{\text {crit }}$. The same scaling in $X$ arises from the basic wave velocity in $E$ in Section 3, while (7.1) itself indicates that $\theta=\theta_{\text {crit }}$ gives the shortest time to breakdown. In addition, the comparison concerning $Z$-scales referred to in the previous paragraph suggests more precisely weighing $\beta \Omega^{-1 / 2} Z$ against $\alpha^{-3 / 2}\left|3 \alpha^{4}-1\right|^{1 / 2} \exp (-2 \alpha T)$, in view of the transformations in Sub-Section 3.4. This gives (7.1) again but with extra terms

$$
\frac{1}{4 \alpha} \ln \left|3 \alpha^{4}-1\right|-\frac{1}{2 \alpha} \ln \beta-\frac{3}{4 \alpha} \ln \alpha,
$$

on the right-hand side. The first term here in particular tends to exaggerate the speed of breakdown for the $\theta=\theta_{\text {crit }}$ growing wave since then $3 \alpha^{4} \rightarrow 1$, from Sub-Section 3.4 .

In contrast, the breakdown to stage 2 for two-dimensional or $\theta<\theta_{\text {crit }}$ nonlinear waves occurs at a time $T$ of order $(3 / 4 \alpha) \ln \Omega$ (from [9]) generally, later than in the three-dimensional $\theta>\theta_{\text {crit }}$ case (7.1).

The subsequent stage 2 arising downstream when the typical time (7.1) is reached has the same double structure I, II as in stage 1 but is fully nonlinear. In the outer region II, where $Y=\Omega^{1 / 2} \hat{y}$, the flow features are now nonlinear in the form

$$
\begin{gathered}
{[U, V, W]=\left[\Omega^{1 / 2} U^{\prime}, \Omega^{3 / 2} V^{\prime}, \Omega^{1 / 2} W^{\prime}\right]\left(X_{0}, \hat{y}, Z_{0}, T_{0}, \ldots\right)+\ldots,} \\
{[P, A]=\left[\Omega P^{\prime}, \Omega^{1 / 2} A^{\prime}\right]\left(X_{0}, Z_{0}, T_{0}, \ldots\right)+\ldots}
\end{gathered}
$$


subject again to the multiple scalings in $X, Z, T$ in (3.1). Hence inviscid unsteady three-dimensional mechanics dominate here, with (1.1) yielding the controlling equations

$$
\begin{gathered}
\partial U^{\prime} / \partial X_{0}+\partial V^{\prime} / \partial \hat{y}+\partial W^{\prime} / \partial Z_{0}=0 \\
\partial U^{\prime} / \partial T_{0}+U^{\prime} \partial U^{\prime} / \partial X_{0}+V^{\prime} \partial U^{\prime} / \partial \hat{y}+W^{\prime} \partial U^{\prime} / \partial Z_{0}=-\partial P^{\prime} / \partial X_{0} \\
\partial W / \partial T_{0}+U^{\prime} \partial W^{\prime} / \partial X_{0}+V^{\prime} \partial W^{\prime} / \partial \hat{y}+W^{\prime} \partial W^{\prime} / \partial Z_{0}=-\partial P^{\prime} / \partial Z_{0}
\end{gathered}
$$

where, here and subsequently, the dependence on the slower scales can be suppressed. The appropriate boundary conditions are:

$$
\begin{aligned}
& U^{\prime} \sim \hat{y}+A^{\prime}\left(X_{0}, Z_{0}, T_{0}\right), W^{\prime} \rightarrow 0, \quad \text { as } \hat{y} \rightarrow \infty, \\
& \text { the } P^{\prime}-A^{\prime} \text { law, } \\
& V^{\prime} \rightarrow 0 \text { as } \hat{y} \rightarrow 0+,
\end{aligned}
$$

from (1.1) and from a typical inviscid assumption about the viscous sublayer at the surface, described below; in (7.5e) the pressure-displacement interaction law is as in (1.1) with $P^{\prime}, A^{\prime}, X_{0}, Z_{0}, T_{0}$ replacing $P, A, X, Z, T$ in turn. The solution of (7.5) induces nonzero unsteady slip velocities, say

$$
U_{e}^{\prime}=U^{\prime}\left(X_{0}, O+, Z_{0}, T_{0}\right), \quad W_{e}^{\prime}=W^{\prime}\left(X_{0}, O+, Z_{0}, T_{0}\right),
$$

in general, at the approach to the solid surface. These are related to the unknown pressure $P^{\prime}$ in the traditional way. The corresponding surface sublayer $I$ then has

$$
[U, V, W]=\left[\Omega^{1 / 2} U^{\prime \prime}, \Omega^{1 / 2} V^{\prime \prime}, \Omega^{1 / 2} W^{\prime \prime}\right]\left(X_{0}, \tilde{y}, Z_{0}, T_{0}, \ldots\right)+\ldots,
$$

with $Y=\Omega^{-1 / 2} \tilde{y}$, so that from (1.1) the motion in the sublayer is governed by the full unsteady three-dimensional boundary-layer equations,

$$
\begin{gathered}
\partial U^{\prime \prime} / \partial X_{0}+\partial V^{\prime \prime} / \partial \tilde{y}+\partial W^{\prime \prime} / \partial Z_{0}=0 \\
\partial U^{\prime \prime} / \partial T_{0}+U^{\prime \prime} \partial U^{\prime \prime} / \partial X_{0}+V^{\prime \prime} \partial U^{\prime \prime} / \partial \tilde{y}+W^{\prime \prime} \partial U^{\prime \prime} / \partial Z_{0} \\
=-\partial P^{\prime} / \partial X_{0}+\partial^{2} U^{\prime \prime} / \partial \tilde{y}^{2} \\
\partial W^{\prime \prime} / \partial T_{0}+U^{\prime \prime} \partial W^{\prime \prime} / \partial X+V^{\prime \prime} \partial W^{\prime \prime} / \partial \tilde{y}+W^{\prime \prime} \partial W^{\prime \prime} / \partial Z_{0} \\
=-\partial P^{\prime} / \partial Z_{0}+\partial^{2} W^{\prime \prime} / \partial \tilde{y}^{2} .
\end{gathered}
$$

The boundary conditions on $(77 \mathrm{a}-\mathrm{c})$ are classical ones, however, in that

$$
\begin{aligned}
& U^{\prime \prime} \rightarrow U_{c}^{\prime}, \quad W^{\prime \prime} \rightarrow W_{e}^{\prime} \quad \text { as } \quad \tilde{y} \rightarrow \infty, \\
& \left(U^{\prime \prime}, V^{\prime \prime}, W^{\prime \prime}\right)=(0,0,0) \quad \text { at } \quad \tilde{y}=0,
\end{aligned}
$$

to reduce the induced slip velocities, determined by region II, to zero at the surface.

Several matters arise immediately. First, the nonlinear equations of motion (7.5) in the inviscid region I form the three-dimensional generalization of the Benjamin-Ono equation (2.1) holding for planar flows. Unfortunately in the analytical sense, the three-dimensional case (7.5) is much more difficult as it does not admit a simple general solution $[37,47]$, unlike in the two-dimensional 
case where $U^{\prime} \equiv y+A^{\prime}$, which leads to (2.1). Only special solutions can be found analytically. Moreover, but of less significance, the decay to the outer boundary conditions (7.5d) is algebraic, as in the full system (1.1). Second, the inviscid problem (7.5) nevertheless seems sufficient to fix the unknown pressure $P^{\prime}$ and displacement $-A^{\prime}$, for suitable starting or other conditions, as well as determining the induced slip velocities $(7.5 \mathrm{~g})$. Third, and most significantly, the above assumes that the classical unsteady sub-boundary-layer flow in region I remains attached. The likelihood, however, is that it does not remain so, and instead it admits a blow-up singularity of its displacement within a finite time $T_{0}$, possibly in a quasi-planar fashion as in $[8,9]$ or of a fully three-dimensional nature. The singularity provides a burst of vorticity from the sublayer I into the lower reaches of region II. This sublayer burst, and especially any later ones provoked, as well as certain secondary instabilities, can occur in an apparently erratic manner just as in the planar-flow case, and despite the mainly inviscid nature of such bursts they do tend to raise substantial doubts about how long the rather simplistic inviscid description (7.5) continues to hold good. Nevertheless, it seems not too unreasonable to argue that the inviscid three-dimensional problem (7.5) merits further study in its own right (with or without the extra $u_{e}, w_{e}$ effects from (1.1)), as a preliminary step, at least until more is understood about the bursting process: on the latter, see also the suggestion in [30]. Next, we note that the above account (7.3)-(7.7) and comments concerning stage 2 apply to the development of each of the individual spikes predicted in the previous section. The overall flowfield is therefore quite a complicated one, rich in structure. The control of the overall strength of the spikes (to which (7.5)-(7.7) match as an initial condition) is a viscous control, of course, by continuation of the viscous control observed in Section 5. Finally here, and as a contrast with the comments of the previous three sections, the stage-2 structure in (7.5), (7.7) can be regarded instead as set up by the abrupt intrusion into the motion (1.1) of a sizeable high-frequency disturbance, of pressure amplitude given in (7.4). Such a non-linear disturbance, whether maintained by forcing or imposed as an initial condition, therefore by-passes the earlier stage 1 of natural downstream development of a free initially small disturbance. The alternative of the abrupt by-pass, which can model the action of sizeable free-stream turbulence on the boundary layer for example, adds to the value of studying the three-dimensional flow structure in stage 2 .

If we continue the argument concerning free disturbances still further, as has been done in the planar case $[8,9]$, a still more nonlinear stage is encountered even further downstream. This is an Euler stage, nominally for effective frequencies $\Omega$ as large as $O\left(\operatorname{Re}^{1 / 4}\right)$. It too can be reached by the bypass mechanism just mentioned. It corresponds to the time scale $t$ being much shorter than in (1.2), namely $t \sim \mathrm{Re}^{-1 / 2}$, with the corresponding $x, z$ scales then both shrinking to the order $\mathrm{Re}^{-1 / 2}$ also, from (3.1). The whole triple-deck in fact contracts to dimensions $O\left(\mathrm{Re}^{-1 / 2}\right)$ in $x, y, z$, with the outer zone II above then filling the boundary layer, while the viscous sublayer thickness decreases to $O\left(\mathrm{Re}^{-3 / 4}\right)$, much as in the planar case. So the developing, nonlinear, TollmienSchlichting disturbance (or the alternative of the abrupt strong by-pass disturbance into the Euler stage) now becomes controlled by the three-dimensional 


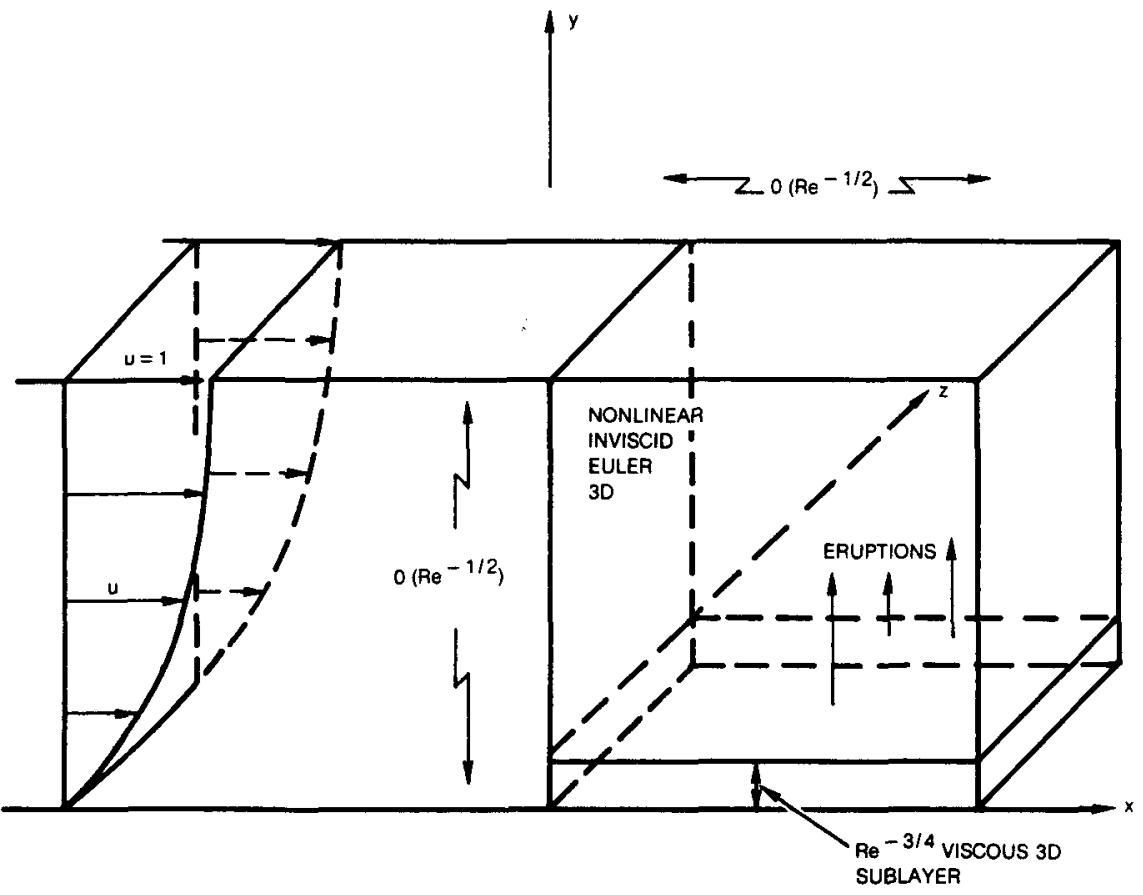

Figure 9. Picture of the later, Euler, stage for even larger three-dimensional disturbances (see Section 7), as implied by the behaviour in the earlier stages 1,2 of the triple-deck.

unsteady Euler equations

$$
\begin{gathered}
\partial u / \partial x_{E}+\partial v / \partial y_{E}+\partial w / \partial z_{E}=0 \\
\partial u / \partial t_{E}+u \partial u / \partial x_{E}+v \partial u / \partial y_{E}+w \partial u / \partial z_{E}=-\partial p / \partial x_{E} \\
\partial v / \partial t_{E}+u \partial v / \partial x_{E}+v \partial v / \partial y_{E}+w \partial v / \partial z_{E}=-\partial p / \partial y_{E} \\
\partial w / \partial t_{E}+u \partial w / \partial x_{E}+v \partial w / \partial y_{E}+w \partial w / \partial x_{E}=-\partial p / \partial z_{e}
\end{gathered}
$$

where $u, v, w, p$ are generally $O(1)$ and the spatial and temporal scales $(x, y, z, t)=\operatorname{Re}^{-1 / 2}\left(x_{E}, y_{E}, z_{E}, t_{E}\right)$ are all much shortened: Fig. 9. The boundary conditions include the merging with the free stream for large $y_{E}$ and tangential flow for small $y_{E}$.

Again, however, this makes the rather bold/gross/simple-minded assumption that the smaller-scale $O\left(\mathrm{Re}^{-3 / 4}\right)$ viscous sublayer, still controlled by (7.7) in effect, remains attached, or at least negligible, and it ignores the likelihood of vorticity bursting, as in stage 2 . The sublayer bursting is clearly of some importance not only theoretically but also experimentally, in transitional and turbulent boundary layers: see $[9,25]$. Granted such doubts about the longrange validity, the three-dimensional unsteady Euler problem (7.8), of which (7.5) is the thin-layer version, would seem nevertheless to be fairly relevant to the larger-scale features of this highly nonlinear stage, and it may be hoped that the finer-scale effects of the sublayer bursting may be accommodated rationaliy in future studies. One intriguing aspect of the Euler flow (7.8) we 
note is that for certain three-dimensional configurations it can itself yield a blow-up or change of scale, in a finite-time singularity: [31]. That blow-up however is for a very special type of flow, near a symmetry line, and is rather open to question, although there may be some useful connection here with the experimental observations of $[1,32]$. See also recent work in $[35,36,39$ 41, 45, 46].

Concerning the more general case of Section 6, there is again exponential growth in amplitude but usually at a lower rate than in the special case and without significant spatial focussing, for periodic flows. Consequently the new stage 2 is encountered later than for the special case. This tends to underline the importance of the special case.

\$8. Further comments. Our additional comments, discussing the main theoretical properties in the paper, the relation with experimental findings, and the further research implied, are presented in (a)-(f) below.

(a) A major finding of the high-frequency analysis in Sections 3-5, for the first nonlinear stage 1 , is the enhanced nonlinear growth of three-dimensional oblique-wave disturbances at angles $\theta$ from the main stream exceeding $\theta_{\text {crit }} \approx 54.7^{\circ}$ in (3.28). This strong three-dimensional growth (Section 5) is accompanied by a certain spikiness and random-looking behaviour in the flow solution as the time and the distance downstream increase, according to the solutions and their spectra in Section 4. The growth is indeed at its fastest exactly at the critical angle $\theta_{\text {crit }}$, according to Section 7 , even though in general it is less violent than that of [34] which however corresponds to quite different input.

(b) The governing equations ( $4.2 \pm$ ) are examples of the Ginzburg-Landau equation, which has been studied in other contexts by numerous authors, e.g., [19-21]. As far as we know, however, the particular case of interest, (4.2 \pm , have not been covered previously, although the limiting forms given in Section 5 above and in [9] would appear to be somewhat relevant to the computations in [19]. The problems addressed in that last reference are slight deviations from (4.2 $)$ and the question of the large-time effect of such deviations is of some mathematical interest, if no more.

(c) Physically, it is interesting first to reconsider the group velocities $C_{g X}$, $C_{g z}$ given in Sub-Section 3.3 and Fig. 3. As would be expected, the cross-flow group velocity $C_{g Z}$ has its maximum value, and the streamwise velocity $C_{g X}$ its minimum, exactly at the critical oblique angle $\theta=\theta_{\text {crit }}$ in (3.28). This tends to add more weight to the view that in a sense single three-dimensional disturbance packets travelling at the critical angle are potentially dangerous. The character of these group velocities, incidentally, agrees broadly with the linear threedimensional calculations in [14]. Also physically, we observe from Section 3 that the mean streamwise- and cross-flow-shear stresses at the surface, $\tau_{X} \equiv$ $\partial U / \partial Y(X, 0, Z, T)$ and $\tau_{Z} \equiv \partial W / \partial Y(X, 0, Z, T)$, are greatly increased from their original steady-laminar-flow values, being given by

$$
\left(\tau_{X}-1, \tau_{Z}\right) \propto\left|P_{01}\right|^{2}
$$

(from (3.8a) and the associated solution for $W_{1 M}$ ) and so increasing fast downstream, in view of Section 5. Again, the oscillations about these mean values 
are larger by an order of magnitude than the mean values themselves, from Sub-Section 3.1. These features are reminiscent of the experimentally observed behaviour in transitional or turbulent boundary layers; see earlier references.

(d) Further downstream, stage 2 can soon be reached, followed by the Euler stage. These are discussed more fully in the previous Section 7 and here we would emphasize only the following points. First, it is encouraging for the rational theory to find the initially linear Tollmien-Schlichting disturbance being transformed nonlinearly, via stages 1, 2, into the strong Euler type of disturbance, which has been considered by other authors using a direct appeal to experimental observations. A second but related point is that theoretically both the growing free disturbance and the by-pass kind produced by abrupt high-frequency forcing can end up at the same stage (Euler); this seems just as in experiments on turbine blades and airfoils, where the turbulent flows resulting downstream of a gradual or a fast transition are virtually identical: see $e . g .,[22]$. Third, we note the overall viscous control of the free-disturbance energy, in Sections 5, 6 and the likelihood of sublayer bursting in stage 2 and the Euler stage, likewise due to viscous effects. It is quite often argued that nonlinear flows such as the Euler kind respond so fast that viscosity cannot exert substantial influence: that is simply not so, from the above. There is in fact a very promising connection between the rational theory and the modelling and experiments described in [25], with regards to the important effects of the viscous sublayer vorticity bursts, further work on which is described in $[35,36,39-41,45,46]$.

(e) Considering further some of the experiments on boundary-layer transition, we return first to the theoretical early stage 1 . Some measure of agreement exists there between the theoretical prediction, of significant three-dimensional effects at oblique angles of $54.7^{\circ}$ (from (3.28)) and greater, and the recent experimental findings of such effects for gradually amplifying disturbances at angles of about $55^{\circ}-60^{\circ}[3,5]$. This may be fortuitous of course, since the former addresses a single nonlinearly growing wave, whereas the latter tend to start with two or more rather tuned waves and an approximately planar wave from which three-dimensional growth then develops downstream. Indeed, alternative physically-motivated explanations are given in $[6,7]$, based on the nonlinear interaction of several waves, e.g., in a resonant triad, and/or on the growth of three-dimensional sub-harmonics downstream, and these agree to a certain extent with experiment. They are also not implausible theoretically, $c f$. the self-consistency relative to the Navier-Stokes equations established rationally in [23]. More recent rational theory on the Klebanoff-type experimental situation $[\mathbf{4 8}]$ is given in $[\mathbf{3 4}, \mathbf{3 6}]$, which show good agreement with experiments at the weakly and the strongly nonlinear stages. In addition, the present work would suggest the desirability of a controlled experiment measuring the nonlinear growth of single three-dimensional wave packets at various oblique angles, to compare with the critical values (3.28). Second, and as mentioned in (d) above, there is a qualitative agreement with experiment and modelling as regards the later stages (2, Euler) of nonlinear development through transition, much as in the planar-flow account in [9], although the three-dimensionality here (Section 5,6 ) does add significantly to the possible sources of strong growth, secondary instability, bursting and apparently erratic behaviour present. 
(f) In view of (e) above and the experimental considerations, it is felt that attempts to enlarge the rational theory further would be very worthwhile. Further research is needed in particular on the following aspects: mode interaction, where two or more nonlinear waves of the kind studied in this paper are present and the issue then arises of which mode predominates, $c f$. the powerful interactions studied in $[34,36]$; resonant-triad response $[7,23]$, which is also a potentially powerful special example of such mode interaction; the nonlinear modes at exactly $\theta=\theta_{\text {crit }}$, which can be the fastest-growing ones (Section 6) and involve a crossover from the case $(4.2+)$ to the case $(4.2-)$; the fully three-dimensional properties of (3.27); slow variation of the wavenumbers $\alpha, \beta$; the more nonlinear response during stage 2 , including the sublayer eruptions then; computational properties of the full three-dimensional triple-deck problem (1.1), its susceptibility to short-scale Rayleigh instability [27] and its relation to the stages 1,2 at high frequency; the stronger Euler stage (Section 6); finite-Reynolds-number computations based on the theory; further investigating the relevance to/of the fascinating theoretical suggestions in $[6,7,31,33]$; and the relation with turbulence-modelling and with large-eddy simulation. The many applications possible to other starting flows such as three-dimensional basic boundary layers and duct flows, and to incorporate free-stream disturbances, wall roughness, section and other laminar-flow control for instance, have also to be pursued fully.

Thanks are due to Drs. M. J. Werle, J. E. Carter, M. Blair, S. E. Melnik and R. E. Whitehead for their comments and interest in this research. This work (see also [47]) was supported by the Office of Naval Research through the offices of Dr. S. E. Whitehead, by the independent program of United Technologies Research Center, and by the Science and Engineering Research Council. Referees' comments are gratefully acknowledged.

Appendix A. Further Details Concerning the Expansions In Section 3. The governing equations resulting from substitution of (3.3) into (1.1) are as follows.

$$
\left.\begin{array}{l}
\text { Continuity: } L\left(U_{0}, V_{0}, W_{0}\right)=0 \\
\begin{array}{rl}
L\left(U_{1}, V_{1}, W_{1}\right) & +\partial U_{0} / \partial X_{1}+\partial W_{0} / \partial Z_{1}=0 \\
L\left(U_{2}, V_{2}, W_{2}\right) & +\left(\partial U_{0} / \partial X_{2}+\partial U_{1} / \partial X_{1}\right) \\
& +\left(\partial W_{0} / \partial Z_{2}+\partial W_{1} / \partial Z_{1}\right)=0
\end{array}
\end{array}\right\}
$$

where $L(u, v, w) \equiv \partial u / \partial X_{0}+\partial v / \partial \tilde{y}+\partial w / \partial Z_{0}$ defines the operator $L$.

$X$-momentum: $\quad M\left(U_{0}, P_{0}\right)=0$,

$$
\begin{aligned}
M\left(U_{1}, P_{1}\right)= & \partial U_{0} / \partial T_{1}+\left(U_{0} \partial U_{0} / \partial X_{0}+V_{0} \partial U_{0} / \partial \tilde{y}+W_{0} \partial U_{0} / \partial Z_{0}\right) \\
& +\partial P_{0} / \partial X_{1} \\
M\left(U_{2}, P_{2}\right)= & \left(\partial U_{0} / \partial T_{2}+\partial U_{1} / \partial T_{1}\right)+\left(U_{0} \partial U_{1} / \partial X_{0}+U_{1} \partial U_{0} / \partial X_{0}\right. \\
& +U_{0} \partial U_{0} / \partial X_{1}+V_{0} \partial U_{1} / \partial \tilde{y}+V_{1} \partial U_{0} / \partial \tilde{y}+W_{0} \partial U_{1} / \partial Z_{0} \\
& \left.+W_{1} \partial U_{0} / \partial Z_{0}+W_{0} \partial U_{0} / \partial Z_{1}\right)+\partial P_{0} / \partial X_{2}+\partial P_{1} / \partial X_{1}
\end{aligned}
$$

where $M(u, p) \equiv \partial^{2} u / \partial \tilde{y}^{2}-\partial u / \partial T_{0}-\partial p / \partial X_{0}$. 
Z-momentum: $\quad N\left(W_{0}, P_{0}\right)=0$,

$$
\left.\begin{array}{rl}
N\left(W_{1}, P_{1}\right)= & \partial W_{0} / \partial T_{1}+\left(U_{0} \partial W_{0} / \partial X_{0}+V_{0} \partial W_{0} / \partial \tilde{y}+W_{0} \partial W_{0} / \partial Z_{0}\right) \\
& +\partial P_{0} / \partial Z_{1}, \\
N\left(W_{2}, P_{2}\right)= & \left(\partial W_{0} / \partial T_{2}+\partial W_{1} / \partial T_{1}\right)+\left(U_{0} \partial W_{1} / \partial X_{0}+U_{1} \partial W_{0} / \partial X_{0}\right. \\
& +U_{0} \partial W_{0} / \partial X_{1}+V_{0} \partial W_{1} / \partial \tilde{y}+V_{1} \partial W_{0} / \partial \tilde{y}+W_{0} \partial W_{1} / \partial Z_{0} \\
& \left.+W_{1} \partial W_{0} / \partial Z_{0}+W_{0} \partial W_{0} / \partial Z_{1}\right)+\partial P_{0} / \partial Z_{2}+\partial P_{1} / \partial Z_{1},
\end{array}\right\}
$$

where $N(W, p) \equiv \partial^{2} w / \partial \tilde{y}^{2}-\partial w / \partial T_{0}-\partial p / \partial Z_{0}$. These yield the solutions quoted in (3.6a) $-(3.11 \mathrm{~b})$ when $(3.4 \mathrm{a}-\mathrm{c})$ are used.

Similarly, substitution of (3.12) into (1.1) produces the successive equations:

$\hat{L}\left(\hat{U}_{0}, \hat{V}_{0}, \hat{W}_{0}\right)=0$,

$\hat{L}\left(\hat{U}_{1}, \hat{V}_{1}, \hat{W}_{1}\right)+\partial \hat{U}_{0} / \partial X_{1}+\partial \hat{W}_{0} / \partial Z_{1}=0$,

$\hat{L}\left(\hat{U}_{2}, \hat{V}_{2}, \hat{W}_{2}\right)+\partial \hat{U}_{0} / \partial X_{2}+\partial \hat{U}_{1} / \partial X_{1}+\partial \hat{W}_{0} / \partial Z_{2}+\partial \hat{W}_{1} / \partial Z_{1}=0$

$$
\left.\begin{array}{l}
\hat{M}\left(\hat{U}_{0}, \hat{V}_{0}, \hat{P}_{0}\right)=0 \\
\hat{M}\left(\hat{U}_{1}, \hat{V}_{1}, \hat{P}_{1}\right)+\partial \hat{U}_{0} / \partial T_{1}+\hat{y} \partial \hat{U}_{0} / \partial X_{1}+\left(\hat{U}_{0} \partial \hat{U}_{0} / \partial X_{0}\right. \\
\left.\quad+\hat{V}_{0} \partial \hat{U}_{0} / \partial \hat{y}+\hat{W}_{0} \partial \hat{U}_{0} / \partial Z_{0}\right)=-\partial P_{0} / \partial X_{1} \\
\hat{M}\left(\hat{U}_{2}, \hat{V}_{2}, \hat{P}_{2}\right)+\left(\partial \hat{U}_{0} / \partial T_{2}+\partial \hat{U}_{1} / \partial T_{1}\right)+\hat{y}\left(\partial \hat{U}_{0} / \partial X_{2}+\partial U_{1} / \partial X_{1}\right) \\
\quad+\left(\hat{U}_{0} \partial \hat{U}_{1} / \partial X_{0}+\hat{U}_{1} \partial \hat{U}_{0} / \partial X_{0}+\hat{U}_{0} \partial \hat{U}_{0} / \partial X_{1}+\hat{V}_{0} \partial \hat{U}_{1} / \partial \hat{y}+\hat{V}_{1} \partial \hat{U}_{0} / \partial \hat{y}\right. \\
\left.\quad+\hat{W}_{0} \partial \hat{U}_{1} / \partial Z_{0}+\hat{W}_{1} \partial \hat{U}_{0} / \partial Z_{0}+\hat{W}_{0} \partial \hat{U}_{0} / \partial Z_{1}\right)=-\left(\partial P_{0} / \partial X_{2}+\partial P_{1} / \partial X_{1}\right)
\end{array}\right\}
$$

$$
\begin{aligned}
& \mid \\
& \}
\end{aligned}
$$

$$
\begin{aligned}
\hat{N}\left(\hat{W}_{2}, \hat{P}_{2}\right)+\left(\partial \hat{W}_{0} / \partial T_{2}+\partial \hat{W}_{1} / \partial T_{1}\right)+\hat{y}\left(\partial \hat{W}_{0} / \partial X_{2}+\partial \hat{W}_{1} / \partial X_{1}\right) \\
+\left(\hat{U}_{0} \partial \hat{W}_{1} / \partial X_{0}+\hat{U}_{1} \partial \hat{W}_{0} / \partial X_{0}+\hat{U}_{0} \partial \hat{W}_{0} / \partial X_{1}+\hat{V}_{0} \partial \hat{W}_{1} / \partial \hat{y}\right. \\
+ \\
\left.+\hat{V}_{1} \partial \hat{W}_{0} / \partial \hat{y}+\hat{W}_{0} \partial \hat{W}_{1} / \partial Z_{0}+\hat{W}_{1} \partial \hat{W}_{0} / \partial Z_{0}+\hat{W}_{0} \partial \hat{W}_{0} / \partial Z_{1}\right) \\
=-\left(\partial P_{0} / \partial Z_{2}+\partial P_{1} / \partial Z_{1}\right),
\end{aligned}
$$

which have the solutions shown in (3.13) and (3.15a)-(3.17a). Here $\hat{L}(u, v, w) \equiv \partial u / \partial X_{0}+\partial v / \partial \hat{y}+\partial w / \partial Z_{0}, \quad \hat{M}(u, v, p) \equiv \partial u / \partial T_{0}+\hat{y} \partial u / \partial X_{0}+v+$ $\partial p / \partial X_{0}, \hat{N}(w, p) \equiv \partial w / \partial T_{0}+\hat{y} \partial w / \partial X_{0}+\partial p / \partial Z_{0}$ define the operators $\hat{L}, \hat{M}, \hat{N}$.

Appendix B. Details of the Mean-Flow Equations Leading To $(3.25 a, b)$ and $(6.1 a, b)$. The mean-flow terms in the third-order momentum equations given 
in Appendix A provide equations for $\hat{U}_{1 M}$ and $\hat{W}_{1 M}$. By considering these mean-flow equations in combination as an equation for $\hat{Q}_{1 M}\left(\equiv \alpha \hat{U}_{1 M}+\beta \hat{W}_{1 M}\right)$ we may find expressions for $\hat{Q}_{1 M}(0)$ and $\hat{Q}_{1 M \hat{y}}$, as follows.

In the frame of reference travelling with the group velocity $\left(c_{1}, c_{2}\right)$ (see (3.22)), we may again replace time derivatives by spatial derivatives. The equation for $\hat{Q}_{1 M}$ then becomes

$$
\begin{aligned}
& {\left[\left(\hat{y}-c_{1}\right) \partial_{X 1}-c_{2} \partial_{Z 1}\right] \hat{Q}_{1 M}} \\
& =-\frac{\beta}{\alpha^{3}}\left[\frac{2}{\alpha \hat{y}-1}+\frac{1}{(\alpha \hat{y}-1)^{2}}\right]\left(\beta \partial_{X 1}-\alpha \partial_{Z 1}\right)\left|p_{01}\right|^{2}-\frac{1}{\alpha^{5}} \frac{\partial\left|p_{01}\right|^{2}}{\partial X_{1}} .
\end{aligned}
$$

The change of variables $\bar{x}=X_{1}+\left(\hat{y}-c_{1}\right) c_{2}^{-1} Z_{1} ; \bar{z}=Z_{1}$, allows the integration of (B1) with respect to $\bar{z}$, and setting $\hat{y}=0$ we obtain $(3.25 \mathrm{c})$, on using the wavenumber relation (3.20).

Differentiating (B1) with respect to $\hat{y}$ and using the mean-flow continuity equation (see Appendix A) leads us to

$$
\left[\left(\hat{y}-c_{1}\right) \partial_{X 1}-c_{2} \partial_{Z 1}\right]^{2} \hat{Q}_{1 M \hat{y}}=\frac{\beta \partial_{X 1}-\alpha \partial_{Z 1}}{\alpha^{2}(\alpha \hat{y}-1)^{2}} \frac{\partial\left|p_{01}\right|^{2}}{\partial Z_{1}}
$$

The above change of variables may again be used, and integrating (B2) twice with respect to $\bar{z}$ then gives $(3.25 \mathrm{~b})$.

In Section 6 we assume that the $X_{1}-Z_{1}$ dependence occurs essentially through $\eta=X_{1}+M Z_{1}$. In this case (3.25c) becomes

$$
\hat{Q}_{1 M}(0)=-\left(\alpha^{2}+\beta^{2}\right)(\alpha+\beta M) \partial_{\eta} \int_{0}^{\infty}\left|p_{01}\left(\eta_{1}+\frac{\hat{c}}{\alpha} \sigma\right)\right|^{2} d \sigma
$$

where $\hat{c}=\alpha\left(c_{1}+M c_{2}\right)$. Integration with respect to $\sigma$ then gives (6.1 a).

Similarly, the left-hand side of $(6.1 \mathrm{~b})$ becomes

$$
M(\beta-\alpha M) \alpha^{-2} \partial_{\eta}^{2} \int_{0}^{\infty} \int_{0}^{\infty}(\alpha \hat{y}-1)^{-4} \sigma\left|p_{01}\left(\eta_{1}-\frac{\sigma}{\alpha}(\alpha \hat{y}-\hat{c})\right)\right|^{2} d \sigma d \hat{y} .
$$

It is helpful to split the range of $\hat{y}$-integration into three, the middle integral being

$$
I_{2}=\int_{\hat{c} / \alpha-\varepsilon}^{\hat{c} / \alpha+\varepsilon}(\alpha \hat{y}-1)^{-2} \hat{Q}_{1 M \hat{y}} d \hat{y}
$$

The two outer integrals may be evaluated using the change of variable $p=$ $\eta_{1}-(\sigma / \alpha)(\alpha \hat{y}-\hat{c})$, to give

$$
I_{1}+I_{3}=M(\beta-\alpha M)\left|p_{01}\right|^{2} \int_{0}^{\infty} \frac{d \hat{y}}{(\alpha \hat{y}-1)^{4}(\alpha \hat{y}-\hat{c})^{2}} .
$$


To evaluate $I_{2}$, let $\hat{y}=\hat{c} / \alpha+\varepsilon s$. Then

$$
I_{2}=M(\beta-\alpha M) \alpha^{-2} \partial_{\eta}^{2} \int_{-1}^{+1} \int_{0}^{\infty} \frac{\varepsilon \sigma\left|p_{01}(\eta-\sigma \varepsilon s)\right|^{2}}{(\alpha \varepsilon s+\hat{c}-1)^{4}} d \sigma d s
$$

Integration with respect to $s$ and $\sigma$ in turn leads formally to

$$
\lim _{\varepsilon \rightarrow 0} I_{2}=\frac{-4 M(\beta-\alpha M)}{\alpha(\hat{c}-1)^{5}} \int_{-\infty}^{\infty} \frac{\left|p_{01}\right|^{2}}{\eta-\eta^{\prime}} d \eta^{\prime}
$$

Equation (6.1b) is thus the result of adding (B5) and (B7).

The presence of a second critical layer becomes apparent in (B5). This is due to the dependence of the mean-flow terms on the slower variables. This layer is assumed to be passive, like that at $\hat{y}=\alpha^{-1}$ (see Sub-Section 3.2). The special case $\hat{c}=1$ is not addressed in the current work.

Appendix C. Demonstrating that the Growing-Spike Solution in (5.3)-(5.6) is itself Stable. Any small perturbation about a given solution $Q=Q_{0}(\eta, \hat{T})=$ $R_{0} \exp \left(i \varphi_{0}\right)$ of the controlling equations $(5.1 \mathrm{a}, \mathrm{b})$ [or $\left.(4.2+)\right]$ is governed by the linear equations

$$
\begin{gathered}
-R_{0} \frac{\partial \varphi_{1}}{\partial \hat{T}}-R_{1} \frac{\partial \varphi_{0}}{\partial \hat{T}}-\frac{\partial^{2} R_{1}}{\partial \eta^{2}}+R_{1}\left(\frac{\partial \varphi_{0}}{\partial \eta}\right)^{2}+2 R_{0}\left(\frac{\partial \varphi_{0}}{\partial \eta}\right)\left(\frac{\partial \varphi_{1}}{\partial \eta}\right)=3 R_{0}^{2} R_{1} \\
-R_{1}+\frac{\partial R_{1}}{\partial \hat{T}}-2\left(\frac{\partial R_{0}}{\partial \eta} \frac{\partial \varphi_{1}}{\partial \eta}+\frac{\partial R_{1}}{\partial \eta} \frac{\partial \varphi_{0}}{\partial \eta}\right)-\left(R_{0} \frac{\partial^{2} \varphi_{1}}{\partial \eta^{2}}+R_{1} \frac{\partial^{2} \varphi_{0}}{\partial \eta^{2}}\right)=0
\end{gathered}
$$

for $R_{1}, \varphi_{1}$, at arbitrary time $\hat{T}$.

At large times, $\left(R_{0}, \varphi_{0}\right)$ becomes the limiting form described in (5.3)-(5.6). Suppose therefore that the perturbation $\left(R_{1}, \varphi_{1}\right)$ has the rather general form

$$
R_{1}=e^{\mu \hat{T}} \hat{R}(\bar{\eta})+\ldots, \quad \varphi_{1}=e^{\hat{\gamma} \hat{T}} \hat{\varphi}(\bar{\eta})+e^{(\hat{\gamma}-8) \hat{T}} \hat{\varphi}(\bar{\eta})+\ldots,
$$

after some order-of-magnitude estimates, where (C1) suggests that the unknown powers in (C3) satisfy $\hat{\gamma}=2+\hat{\mu}$. Then (C2) requires $\hat{\varphi}(\bar{\eta})$ to be constant, leaving $\hat{R}(\bar{\eta})$ governed by the equation

$$
\hat{R}^{\prime \prime}+\left(3 \bar{R}^{2}(\bar{\eta})-4 K_{m}\right) \hat{R}=-\hat{\gamma} \bar{R}(\bar{\eta}) \hat{\varphi} .
$$

Here $\bar{R}(\bar{\eta})$ is given in (5.5), and henceforth we take $K_{m}=1 / 4$ without loss of generality. The aim is to determine the maximum growth rate $\hat{\gamma}$ [or $\hat{\mu}=\hat{\gamma}-2$ ] of the perturbation.

The solution of (C4) bounded for all $\bar{\eta}$ is

$$
\hat{R}(\bar{\eta})=\hat{B} \tanh (\bar{\eta}) \operatorname{sech}(\bar{\eta})+\frac{\hat{\gamma} \hat{\varphi}}{\sqrt{2}}\{\bar{\eta} \tanh (\bar{\eta})-1\} \operatorname{sech}(\bar{\eta})
$$


where the constant $\hat{B}$ is arbitrary. On the other hand, for the correction term $\hat{\varphi}$ in (C3) to have no exponential growth as $|\bar{\eta}| \rightarrow \infty,(\mathrm{C} 2)$ is found to require that

$$
(\hat{\mu}-2) \int_{-\infty}^{\infty} \hat{R}(\bar{\eta}) \operatorname{sech}(\bar{\eta}) d \bar{\eta}=0 .
$$

Substitution of (C5) into (C6) then yields the requirement $\hat{\gamma} \hat{\varphi}(\hat{\mu}-2)=0$. So the maximum growth rate of the perturbation has

$$
\hat{\mu}=2, \quad \hat{\gamma}=4 .
$$

These values coincide with the growth rates of the basic spike solution in (5.3), however. Hence the perturbation is, in relative terms, stable. Further, if the integral on the right-hand side of (5.2) is kept fixed then (C6) is implied again but without its $(\hat{\mu}-2)$ factor shown; this reduces the growth rate still further by requiring in general that $\hat{\mu}=-2, \hat{\gamma}=0$, in view of (C5).

\section{References}

1. P. S. Klebanoff, K. D. Tidstrom and L. M. Sargent. J. Fluid Mech., 12 (1962), 1.

2. I. Wygnanski, M Sokolov and D Friedman. J. Fluid Mech., 78 (1976), 785.

3. Yu. S. Kachanov and V. Ya. Levchenko. J. Fluid Mech., 138 (1984), 209.

4. M. Gaster, Proc. Symp. on Turb. and Chaotic Phen, in Fluids, Kyoto, Japan (ed. T. Tatsumi) (Elsevier, 1984); see also Transition and Turbulence, 95, ed. S. E. Meyer (Academic Press, 1981).

5. W. S. Saric, V. V. Kozlov and V. Ya. Levchenko. AIAA Paper No. 84-0007 (presented January 1984, Reno, Nevada).

6. T. Herbert. AIAA Paper No. 84-0009 (presented January 1984, Reno, Nevada).

7. A. D. D. Craik. J. Fluid Mech., 50 (1971), 393; see also Craik, 1984, Proc. IUTAM Symp. on Lam.-Turb. Transition, Novosibirsk, USSR, (ed. V. V. Kozlov) (Springer-Verlag, 1985).

8. F. T. Smith and O. S. Burggraf. Proc. Roy. Soc. A, 399 (1985), 25.

9. F. T. Smith. Utd. Tech. Res. Center, E. Hartford, Conn., Rept. UTRC85-36, 1985, and J. Fluid Mech., 1986.

10. P. W. Duck. J. Fluid Mech., 160 (1985), 465.

11. F. T. Smith. Proc. Roy. Soc. A, 366 (1979), 91 and A, 368 (1979), 573.

12. M. E. Goldstein. J. Fluid Mech., 154 (1985), 509-530.

13. P. Hall and F. T. Smith. Stud. in Appl. Math., 70 (1984), 91.

14. W. O. Criminale and L. S. G. Kovasnay. J. Fluid Mech., 14 (1962), 59.

15. P. Hall. Proc. Roy. Soc. A, 406 (1986), 93-106.

16. P. A. Stewart and F. T. Smith. Proc. Roy. Soc. A, 409 (1987), 229-248.

17. F. T. Smith, D. Papageorgiou and J. W. Elliott. J. Fluid Mech., 146 (1984).

18. N. Itoh. Trans. Jap. Soc. Aerosp. Sc., 17 (1974), 175.

19. C. S. Bretherton and E. A. Spiegel. Phys. Letters, 96A (1983), 152.

20. H. T. Moon, P. Huerre and L. G. Redekopp. Phys. Rev. Letter, 49 (1982), 48.

21. Y. Kuramoto. Suppl. of Progress of Theor. Phys., No. 64 (1978), 346.

22. D. J. Doorly. D. Phil. Thesis, Univ. of Oxford, 1983; see also: D. J. Doorly and M. L. C. Oldfield, ASME paper 85-GT-112, (1985), presented at ASME Gas Turbine Conf., Houston, Texas, March 1985 (and Trans. ASME Eng. for Power (1986)); and D. J. Doorly, M. L. G. Oldfield and C. T. J. Scrivener, AGARD C.P.P. 390 (1985), presented at AGARD Mtg., Bergen, Norway, May 1985.

23. F. T. Smith and P. A. Stewart. J. Fluid Mech., 179 (1987), 227-252.

24. R. J. Bodonyi and F. T. Smith. Proc. Roy. Soc. A, 375 (1981), 65.

25. J. D. A. Walker and R. K. Scharnhorst. In Recent Advs. in Eng. Sci., ed. G. C. Sih, 541, (Univ. Press, Bethlehem, Penn., 1977); see also Walker \& Abbott, in Turb. in Int. Flows, ed. S. N. B. Murthy, 131 (1977), (Hemisph. Pub. Corp., Wash., 1977).

26. H. Fasel. Proc. Symp. on Turb. \& Chaotic Phen. in Fluids, Kyoto, Japan, ed. T. Tatsumi (Elsevier, 1984). 
27. F. T. Smith and R. J. Bodonyi. Aeron. Jnl. of Roy Aeron. Soc., June/July (1985), 205-212.

28. A. Davey, L. M. Hocking and K. Stewartson. J. Fluid Mech., 63 (1974), 529.

29. P. A. Stewart. Ph.D. Thesis, Univ. of London, in preparation.

30. F. T. Smith. Proc. Symp. on Stability of Time-Dependent and Spatially Varying Flows, NASA Langley Res. Cent., Hampton, VA., Aug. 1985, (Springer-Verlag, 1986) see also United. Tech. Res. Cent., East Hartford, Rept. UTR C85-55.

31. M. E. Stern and N. Paldor. Phys. Fluids, 26 (1983), 906.

32. L. S. G. Kovasznay, H. Komoda and R. B. Vasudiva. Heat Transfer \& Fluid Mech. Inst., ed. F. E. Elles, J. Kauzlarich, C. A. Sleicher and S. Street (Stanford Univ. Press, CA, 1962).

33. D. J. Benney. Proc. Symp. on time-dependent and spatially varying flows, NASA Langley Res. Cent., Hampton, VA, Aug. 1985 (Springer-Verlag, 1986).

34. P. A. Stewart and F. T. Smith. J. Fluid Mech., 244 (1992), 79-100.

35. F. T. Smith. Mathematika 35 (1988), 256-273.

36. F. T. Smith and R. I. Bowles. Proc. Roy Soc. A, 439 (1992), 163.

37. Y. S. Kachanov, O. S. Ryzhov and F. T. Smith. J. Fluid Mech., 251 (1993), 273.

38. D. A. R. Davis. On linear and nonlinear instability in boundary layers with crossflow. Ph.D. Thesis, Univ. of London (1992).

39. J. M. Hoyle. Extensions to the theory of finite-time breakdown of unsteady interactive boundary layers. Ph.D. Thesis, Univ. of London (1992).

40. V. J. Peridier, F. T. Smith and J. D. A. Walker. J. Fluid Mech., 232 (1992), 99-131 \& 133165.

41. J. M. Hoyle, F. T. Smith and J. D. A. Walker. Comput. Phys. Commns., 65 (1991), 151-157.

42. M. E. Goldstein \& L. S. Hultgren. Ann. Rev. Fluid Mech. 21 (1989), 137-166.

43. F. T. Smith and P. Blennerhassett. Proc. Roy. Soc. A, 436 (1992), 585-602.

44. A. P. Rothmayer and F. T. Smith. Trans. A.S.M.E. Conf., Cincinnati, Ohio, June, 1987 (1987).

45. F. T. Smith, D. J. Doorly and A. P. Rothmayer. Proc. Roy. Soc. A, 428 (1990), 255-281.

46. C. S. Smith, J. D. A. Walker, A. H. Haidari and U. Sobrun. Trans. Roy. Soc. A, 336 (1991), 131-175.

47. F. T. Smith. Utd. Techn. Res. Center Rept. 86-10 (1986).

48. P. S. Klebanoff and K. D. Tidstrom. Tech. Notes, Nat. Aero. Space Admin. Wash. D-195, (1959).

Professor F. T. Smith,

Department of Mathematics,

University College London,

Gower Street,

London. WC1E 6BT

Mr. P. A. Stewart,

D.A.M.T.P.,

Silver Street,

Cambridge. CB3 9EW

Dr. R. G. A. Bowles,

Department of Mathematics,

University College London,

Gower Street,

London. WCIE 6BT
76D15: FLUID MECHANICS: Incompressible viscous fluids; Boundary-layer separation.

Received on the 27th of October, 1992. 